\title{
THE UNIQUENESS OF GROUPS OF LYONS TYPE
}

\author{
MICHAEL ASCHBACHER AND YOAV SEGEV
}

We give the first computer free proof of the uniqueness of groups of type $L y$. We also show that certain simpicial complexes associated to the Lyons group $L y$ have the same homotopy type as the Quillen complex for $L y$ at the prime 3; we show this complex is simply connected, and we calculate its homology. Finally we supply simplified proofs of some properties of groups of type $L y$, such as the group order.

A group of type $L y$ is a finite group $G$ possessing an involution $t$ such that $H=C_{G}(t)$ is the covering group of the alternating group $A_{11}$ and $t$ is not weakly closed in $H$ with respect to $G$. We prove

Theorem 1. Up to isomorphism there exists at most one group of type Ly.

Lyons was the first to consider groups of type $L y$ in [7], where he determined the local structure, the group order, and the character table of such groups. Lyons left open the existence and uniqueness of groups of type $L y$.These questions were settled by Sims in [9] using extensive machine computation.

Our proof is based on the theory of uniqueness systems developed in [2]. We apply this theory to the 3-local geometry $\Gamma$ of a group $G$ of type $L y$ and its collinearity graph $\Delta$. We also use $\Delta$ to give a much more elementary derivation of the group order. Given the theory in [2], the most difficult step in the uniqueness proof is to show that the graph $\Delta$ is simply connected in the language of [2].

Associated to any graph $\theta$ is its clique complex $K(\theta)$, which is the simplicial complex whose vertices are the vertices of $\theta$ and whose simplices are the cliques of $\theta$. We define the homology of $\theta$ to be the homology of the topological space of its clique complex, and we say two graphs have the same homotopy type if the homotopy type of the topological spaces of their clique complexes is the same. By Remark 5 in [2], a graph is simply connected if and only if the topological space of its clique complex is simply connected. Finally we recall that the Quillen complex of a finite group $G$ at a prime $p$ is the complex of the poset of elementary abelian $p$-subgroups of $G$ (cf. [8]). We prove

Theorem 2. Let $G$ be a group of type Ly, $\Delta$ the commuting graph on 3-central subgroups of $G$ of order $3, \Lambda$ the commuting graph on subgroups of $G$ of order

Received by the editors September 18, 1990.

1991 Mathematics Subject Classification. Primary 20D05; Secondary 55U05.

This work was partially supported by BSF 88-00164. The first author was partially supported by NSF DMS-8721480 and NSA MDA90-88-H-2032. 
3 , and $\Gamma$ the 3-local geometry of $G$. Then

(1) $\Delta, \Lambda, \Gamma$, and the Quillen complex $\mathscr{A}_{3}(G)$ of $G$ at the prime 3 all have the same homotopy type.

(2) Each of these graphs and complexes is simply connected.

(3) $\widetilde{H}_{i}(\Delta)=0$ for $i \neq 2$, while $\operatorname{dim}\left(H_{2}(\Delta)\right)=531,228,318,624$.

We do not assume any of Lyons's work from [7], but [7] was invaluable in suggesting what to prove. Also our initial analysis in $\S 4$ is much like that of Lyons. It should be added that Lyons's paper is a pleasure to read. While we do not assume any facts about $L y$, we do assume certain facts about McLaughlin's group that are recorded in the first four lemmas of $\S 3$.

\section{SOME RESULTS ON GRAPHS}

In this section $\Delta$ and $\Gamma$ are graphs. (The term graph will always mean undirected graph.) We adopt the terminology and notational conventions of $\S \S 2$ and 3 of [2]. In particular, if $S$ is a set of cycles of $\Delta$ then $\langle S\rangle$ denotes the closure of $S$ in the path groupoid $P(\Delta)$ of $\Delta$, as defined in $\S 2$ of [2]. Recall $\operatorname{Bas}(\Delta)$ is the smallest closed subset of $P(\Delta)$. We write $\mathscr{C}_{n}(\Delta)$ for the closure of all cycles of length at most $n$.

Given a set $X$ and a symmetric relation $R$ on $X$, we can regard $X$ as a graph with edge set $R$. For $x \in X$, we write $R(x)$ for the set of $y$ distinct from $x$ such that $(x, y) \in R$. We say $X$ is $R$-connected if the graph $(X, R)$ is connected.

Recall that for $x \in \Delta, x^{\perp}=\{x\} \cup \Delta(x)$ and a morphism $\phi: \Lambda \rightarrow \Delta$ of graphs is a map of vertices such that $\phi\left(x^{\perp}\right) \subseteq \phi(x)^{\perp}$ for all $x \in \Lambda$. Throughout this section $\phi: \Lambda \rightarrow \Delta$ is assumed to be a morphism of graphs. We extend $\phi$ to a map $\phi: P(\Lambda) \rightarrow P(\Delta)$ via $\phi\left(x_{0} \cdots x_{n}\right)=\phi\left(x_{0}\right) \cdots \phi\left(x_{n}\right)$. Then the extended map $\phi$ is a morphism of groupoids; that is,

Lemma 1.1. Let $p, q$ be paths in $\Lambda$ with $\operatorname{end}(p)=\operatorname{org}(q)$. Then

(1) $\phi\left(p^{-1}\right)=\phi(p)^{-1}$.

(2) $\phi(p q)=\phi(p) \phi(q)$.

Lemma 1.2. Let $S$ be a set of cycles of $\Lambda$. Then

(1) $\phi(\operatorname{Bas}(\Lambda)) \subseteq \operatorname{Bas}(\Delta)$.

(2) $\phi(\langle S\rangle) \subseteq\langle\bar{\phi}(S)\rangle$.

(3) If $p, q$ are paths in $\Lambda$ with $p \sim_{\langle S\rangle} q$ then $\phi(p) \sim_{\langle\phi(S)\rangle} \phi(q)$.

Proof. Let $\sim$ be an invariant equivalence relation on $\Delta$ and define a relation $\simeq$ on $\Lambda$ by $p \simeq q$ if $p$ and $q$ have the same origin and end and $\phi(p) \sim \phi(q)$. Using Lemma 1.1 we check that $\simeq$ is an invariant equivalence relation on $\Lambda$. In particular $\operatorname{ker}(\simeq)$ is closed and consists of the cycles in $\phi^{-1}(\operatorname{ker}(\sim))$. As $\operatorname{ker}(\simeq)$ is closed it contains $\operatorname{Bas}(\Lambda)$, and applying this observation to the basic relation in the role of $\sim$, we get $(1)$. Similarly applying it to $\sim_{\langle\phi(S)\rangle}$ in the role of $\sim$, we get $(2)$.

Finally assume the hypotheses of (3). Then $p q^{-1} \in\langle S\rangle$, so by Lemma 1.1, $\phi(p) \phi(q)^{-1}=\phi\left(p q^{-1}\right) \in \phi(\langle S\rangle) \subseteq\langle\phi(S)\rangle$, so $\phi(p) \sim_{\langle\phi(S)\rangle} \phi(q)$. That is (2) implies (3). 
Lemma 1.3. $\phi\left(\mathscr{C}_{n}(\Lambda)\right) \subseteq \mathscr{C}_{n}(\Delta)$ for each $n$.

Proof. By definition, $\mathscr{C}_{n}(\Lambda)=\langle S\rangle$, where $S$ is the set of all cycles of $\Lambda$ of length at most $n$. But if $p$ is a cycle of length $m$ then $\phi(p)$ is too, so by Lemma 1.2, $\phi\left(\mathscr{C}_{n}(\Lambda)\right)=\phi(\langle S\rangle) \subseteq\langle\phi(S)\rangle \subseteq \mathscr{C}_{n}(\Delta)$.

Lemma 1.4. Define a sequence $R_{0} \subseteq R_{1} \subseteq \cdots$ of symmetric relations on $\Delta$ recursively as follows: $R_{0}$ is the edge set of $\Delta$. Given $R_{i}, R_{i+1}$ is the set of pairs $(x, y)$ such that $y \in \Delta^{2}(x)$ and $\Delta(x, y)$ is $R_{i}$-connected, let $R_{\infty}=\bigcup_{i} R_{i}$ and $p=x_{0} \cdots x_{4}$ a square with $x_{2} \in R_{\infty}\left(x_{0}\right)$. Then $p \in \mathscr{C}_{3}(\Delta)$.

Proof. By hypothesis $x_{2} \in R_{m}\left(x_{0}\right)$ for some $m \geq 1$. We induct on $m$. If $m=$ 1 the result is 3.4 in [2], so take $m>1$. Then by hypothesis there is an $R_{m}$-path $x_{1}=y_{0} \cdots y_{k}=x_{3}$ in $\Delta\left(x_{0}, x_{2}\right)$. As $y_{i+1} \in R_{m}\left(y_{i}\right), p_{i}=x_{0} y_{i} x_{2} y_{i+1} x_{0} \in \mathscr{C}_{3}(\Delta)$ by induction on $m$. Thus as $p$ is in the closure of the paths $p_{i}, 0 \leq i<k$, also $p \in \mathscr{C}_{3}(\Delta)$.

Lemma 1.5. Let $p=x_{0} \cdots x_{5}$ be a pentagon in $\Delta$ such that $x_{0}^{\perp} \cap x_{2}^{\perp} \cap x_{3}^{\perp} \neq \varnothing$. Then $p \in \mathscr{C}_{4}(\Delta)$.

Proof. Let $x \in x_{0}^{\perp} \cap x_{2}^{\perp} \cap x_{3}^{\perp}$. Then $p$ is in the closure of the triangle $x x_{2} x_{3} x$ and the squares $x_{0} x_{1} x_{2} x x_{0}$ and $x_{0} x x_{3} x_{4} x_{0}$.

\section{SOME GROUP THEORETIC PRELIMINARIES}

Lemma 2.1. Let $G$ be a finite group, $t$ an involution in $G, H=C_{G}(t)$, and $T \in \operatorname{Syl}_{2}(H)$. Assume

(1) $H=\left\langle t^{G} \cap H\right\rangle$.

(2) $O(H)=1$.

(3) $\langle t\rangle=Z(T)$.

(4) There is a 4-subgroup $E$ of $G$ with $E^{\sharp} \subseteq t^{G}$.

Then $G$ is a nonabelian simple group.

Proof. First $O(G)=\left\langle C_{O(G)}(e): e \in E^{\sharp}\right\rangle$, so as $O(H)=1$, we have $O(G)=1$.

Let $M$ be a minimal normal subgroup of $G$. As $O(G)=1,|M|$ is even, so as $\langle t\rangle=Z(T), t \in M$. Therefore $H=\left\langle t^{G} \cap H\right\rangle \leq M$. In particular by (3), $T \in \operatorname{Syl}_{2}(M)$, so by a Frattini argument, $G=M N_{G}(T)$. Then as $\langle t\rangle=Z(T)$, $G=M H=M$.

Therefore $G$ is simple, and as $E$ exists, $G$ is not of prime order.

Lemma 2.2. Let $\Omega=\{1, \ldots, 11\}, G=\operatorname{Alt}(\Omega)$, and $\Delta$ the commuting graph on subgroups of $G$ of order 3 generated by a cycle. Then

(1) $\Delta$ is of diameter 2 and simply connected.

(2) Let $M_{11} \cong M \leq G, \theta$ be the set of subgroups of $M$ of order 3 , and $\Lambda$ be the bipartite graph on $\Lambda \cup \theta$ obtained by joining $x \in \Delta$ to $a \in \theta$ if $[x, a]=1$. Then $\Lambda$ is connected.

Proof. For $S \subseteq G$ let $\operatorname{Fix}(S)$ and $\operatorname{Mov}(S)$ be the set of points of $\Omega$ fixed by $S$ and moved by $S$, respectively. Then the map $x \mapsto \operatorname{Mov}(x)$ is a bijection 
between $\Delta$ and 3-subsets of $\Omega$. Let

$$
\Delta_{i}(x)=\{y \in \Delta:|\operatorname{Mov}(y) \cap \operatorname{Fix}(x)|=i\} .
$$

Then by 9-transitivity of $G$ on $\Omega, \Delta_{i}(x), 0 \leq i \leq 3$ are the orbits of $G_{x}$ on $\Delta$. Further $\Delta(x)=\Delta_{3}(x)$ and for all $y \in \Delta,|\operatorname{Fix}(\langle x, y\rangle)| \geq 5$, so $\Delta(x, y) \neq \varnothing$ and hence $\Delta$ has diameter 2 .

Now to prove (1), it remains to show $\Delta$ is simply connected; equivalently by 4.3 in [2], we must show each cycle in $\Delta$ is in $\mathscr{C}_{3}(\Delta)$. As $\Delta$ is of diameter 2 it follows from 3.3 in [2] that it suffices to show each square and pentagon in $\Delta$ is in $\mathscr{C}_{3}(\Delta)$.

Next we observe that if $y \in \Delta_{1}(x)$ then $\Delta(x, y)$ is connected, so in the language of Lemma 1.4, $\Delta_{1}(x) \subseteq R_{1}(x)$. Similarly if $z \in \Delta_{2}(x)$ then $\Delta(x, z)$ is $R_{1}$-connected, so $\Delta^{2}(x) \subseteq R_{2}(x)$. Thus $\mathscr{C}_{4}(\Delta)=\mathscr{C}_{3}(\Delta)$ by Lemma 1.4 .

Finally let $p=x_{0} \cdots x_{5}$ be a pentagon in $\Delta$. Then $\left|\operatorname{Fix}\left(\left\langle x_{0}, x_{2}, x_{3}\right\rangle\right)\right| \geq 4$, so $\Delta\left(x_{0}, x_{2}, x_{3}\right) \neq \varnothing$. Hence Lemma 1.5 completes the proof.

Now to the proof of (2). Recall $M$ is 4-transitive on $\Omega$ and if $a=\langle g\rangle \in \theta$ then $g$ has 3 cycles $g_{i}$ on $\Omega$ of length 3 . Thus $x_{i}=\left\langle g_{i}\right\rangle, 1 \leq i \leq 3$ are the 3 members of $\Lambda(a)$. In particular, $M$ is a group of automorphisms of $\Lambda$, and as $M$ is 3-transitive on $\Omega, M$ is transitive on pairs $(b, x)$ with $b \in \theta, x \in \Delta$, and $x \in \Lambda(b)$. Thus $\Lambda$ is connected if and only if $M=\left\langle M_{a}, M_{x_{1}}\right\rangle$. But this holds as $M_{x_{1}}$ is a maximal subgroup of $M$ that does not contain $M_{a}$.

Lemma 2.3. Let $F$ be a field, $G$ a finite group and $V$ a finite-dimensional faithful $F G$-module with $C_{V}(G)=0$. Then

(1) There exists a largest $F G$-module $U=U(G, V)$ such that $V \leq U$, $C_{U}(G)=0$, and $[U, G] \leq V$.

(2) $\operatorname{dim}\left(H^{1}(G, V)\right)=\operatorname{dim}(U / V)$.

(3) The dual $U^{*}$ of $U$ is the largest $F G$-module $W$ such that $W=[W, G]$ and $W / C_{W}(G) \cong V^{*}$.

(4) The representation of $N_{\mathrm{GL}(V)}(G)$ on $V$ extends to a representation on $U$.

Proof. See 17.11 and 17.12 in [1] for parts (1)-(3). Part (4) holds as $U$ may be regarded as the group of automorphisms of the semidirect product $G V$ centralizing $V$ and $G V / V$; hence $N_{\mathrm{GL}(V)}(G)$ is faithfully represented on $U$ via conjugation.

Lemma 2.4. Let $G \cong A_{6}, V$ a faithful $\mathrm{GF}(3) G$-module of dimension $4, A=$ $N_{\mathrm{GL}(V)}(G)$, and $U=U(G, V)$. Then

(1) $V$ is determined up to isomorphism as a $\mathrm{GF}(3) G$-module, $G$ is absolutely irreducible on $V$, and $A / G \cong D_{8}$.

(2) $\operatorname{dim}\left(H^{1}(G, V)\right)=2$.

(3) $A$ has two orbits on hyperplanes of $U$ containing $V$ with representatives $U_{1}$ and $U_{2}$ such that $N_{A}\left(U_{1}\right) \cong \mathbf{Z}_{2} \times S_{6}$ and $N_{A}\left(U_{2}\right) \cong \mathbf{Z}_{2} \times M_{10}$.

Proof. The first two statements in part (1) are well known. For the third observe that the semidirect product $S=G V$ is a local subgroup of $H=U_{4}(3)$ and 
if $B=\operatorname{Aut}(H)$ then $B / H \cong D_{8}, B=H N_{B}(S)$, and $V$ is self-centralizing in $N_{B}(S)=D$. Thus $D / V \leq A$ with $D / G V \cong D_{8}$. Further as $G$ is absolutely irreducible on $V,\left|C_{A}(G)\right|=2$, while $|\operatorname{Out}(G)|=4$, so indeed $D / V=A$.

Next $G$ has two conjugacy classes of subgroups $L^{G}$ and $K^{G}$ with $L \cong K \cong$ $A_{5}, L=K^{a}$ for some $a \in A$, and $V \cong\left[V_{I}, G\right] / C_{V_{I}}(G)$, where $V_{I}$ is the permutation module for $G$ over $F$ on the cosets of $I=L$ or $K$. Notice, however, that $U_{L}=V_{L} / C_{V_{L}}(G)$ is not isomorphic to $U_{K}=V_{K} / C_{V_{K}}(G)$ as $L$ fixes a point in the former but not the latter. Thus $\operatorname{dim}\left(H^{1}(G, V)\right) \geq 2$.

Observe next that $\operatorname{dim}\left(H^{1}(L, V)\right)=1$. This is because $W / C_{W}(L)=$ $U(L, V)$, where $W$ is the six-dimensional permutation module for $L$, since a $D_{10}$-subgroup of $L$ fixes a point in $U-V$ for any extension $U$ of $V$ by the trivial $L$-module.

Thus if $Z$ is an $F G$-module with $C_{Z}(G)=0$ and $[Z, G]=V$ and $\operatorname{dim}(Z / V)>2$, then there exists points $X, Y$ of $Z$ fixed by $L, K$, respectively with $V+X=V+Y$. Thus $V+X \cong U_{L} \cong U_{k}$, a contradiction. So (2) is established.

Finally by Lemma 2.3, the action of $A=N_{\mathrm{GL}(V)}(G)$ extends to $U$. Let $e \in A$ induce scalar action on $V$. Then $\langle e\rangle=C_{A}(G)$ and as $C_{U}(G)=0$, $C_{U}(e)=0$, so $A / G \cong D_{8}$ is faithful on $U / V$ and hence has two orbits on the points of $U / V$ with representatives $U_{i} / V, i=1,2$. We may pick $U_{1} \cong U_{L}$, so $N_{A}\left(U_{1}\right) \cong \mathrm{Z}_{2} \times S_{6}$. As $U_{2}$ is the restriction to $G$ of the five-dimensional irreducible for $M_{11}$ discussed in the next lemma, $N_{A}\left(U_{2}\right) \cong \mathrm{Z}_{2} \times M_{10}$.

Lemma 2.5. Let $G \cong \mathrm{Z}_{2} \times M_{11}, M=[G, G], V$ a faithful five-dimensional $\mathrm{GF}(3) G$-module, and $K=G V$ the semidirect product. Then

(1) $V$ is determined up to isomorphism as a $\mathrm{GF}(3) M$-module, so $K$ is determined up to isomorphism.

(2) $M$ has two orbits on the points of $V$ of length 11 and 110 .

(3) If $x$ is a point in the orbit of length 11 then $K_{x}=\operatorname{Aut}\left(K_{x}\right)$.

Proof. For (1) see James [5]. Then (2) is an easy calculation. Moreover $M_{x} \cong$ $M_{10}$ and $E=E\left(M_{x}\right) \cong A_{6}$ is indecomposable on $V$, so $V$ is isomorphic to the dual of the module $U_{2}$ of Lemma $2.4(3)$ as an $E$-module. Thus $N_{\mathrm{GL}(V)}(E)=$ $G_{x}$ by Lemma $2.4(3)$. Further as $V=[V, Z(G)], H^{1}\left(G_{x}, V\right)=0$. Then (3) follows from these two facts.

\section{GROUPS OF TYPE $M c$}

A finite group $G$ is of type $M c$ if $G$ possesses an involution $t$ such that $C_{G}(t)$ is the covering group of $A_{8}$ and $t$ is not weakly closed in $C_{G}(t)$ with respect to $G$.

In this section we assume $G$ is of type $M c$ and let $\widehat{G}=\operatorname{Aut}(G)$ and $H=$ $C_{G}(t)$. In Lemmas 3.1-3.4 we record the facts we will be assuming about $G$. Most of them are well known (cf. [10,6, 4]).

Lemma 3.1. (1) Up to isomorphism there is a unique group of type $M c$.

(2) $G$ has one class of involutions. 
(3) $|\operatorname{Out}(G)|=2, G$ is transitive on involutions in $\widehat{G}-G$, and $C_{G}(s) \cong M_{11}$ for each such involution.

(4) The Schur multiplier of $G$ has order 1 or 3.

(5) $|G|=2^{7} \cdot 3^{6} \cdot 5^{3} \cdot 7 \cdot 11$.

(6) Elements of odd order inverted by involutions in $G$ are of order 3 or 5.

Let $\Delta$ be the commuting graph on the 3-central subgroups of $G$ of order 3; we also write $\Delta$ for the set of vertices of this graph.

Lemma 3.2. (1) $G$ has two classes of elements of order 3 .

(2) $G$ is transitive on $\Delta$ and for $x \in \Delta, \widehat{G}_{x}$ is the split extension of $O_{3}\left(G_{x}\right)=$ $R \cong 3^{1+4}$ by $\mathbf{Z}_{2} /\left(\mathbf{Z}_{4} * \mathrm{SL}_{2}(5)\right)$.

(3) Let $T \in \operatorname{Syl}_{3}\left(G_{x}\right)$ and $A=\langle T \cap \Delta\rangle$. Then $A=J(T) \cong E_{81}$ and $N_{\widehat{G}}(A)$ is the split extension of $A$ by $\mathbf{Z}_{2} \times M_{10}$ with $T \cap \Delta$ of order 10 and $A$ inverted by an involution in $\widehat{G}-G$.

(4) $T=R A$ and all elements of order 3 in $T$ are contained in $R$ or $A$.

(5) $x$ is weakly closed in $R$ with respect to $G$.

(6) $G_{x}$ has five orbits $\Delta_{i}(x), 0 \leq i \leq 4$, on $\Delta$, with $\Delta_{0}(x)=\{x\}, \Delta_{1}(x)=$ $\Delta(x)$, and $\langle x, y\rangle \cong \mathrm{SL}_{2}(3), \mathrm{SL}_{2}(5), \mathrm{Z}_{3} / 5^{1+2}$ for $y \in \Delta_{i}(x)$ and $i=2,3,4$, respectively.

(7) $G_{x}$ is transitive on involutions $s \in \widehat{G}_{x}-G_{x}$ and $G_{x, s} \cong M_{9}$.

Lemma 3.3. (1) $G$ has two classes of subgroups of order 5 with representatives $X$ and $X_{2}$.

(2) $N_{G}(X)$ is the split extension of $F^{*}\left(N_{G}(X)\right)=O_{5}\left(N_{G}(X)\right)=P \cong 5^{1+2}$ by $\mathbf{Z}_{8} / Z_{3}$.

(3) $N_{\widehat{G}}\left(X_{2}\right) \leq N_{\widehat{G}}(X)$ with $C_{G}\left(X_{2}\right)=X X_{2}$.

(4) $X$ is weakly closed in $N_{G}(X)$ with respect to $G$.

(5) There is an involution $s \in N_{\widehat{G}}(X)-G$ with $C(s) \cap N_{\widehat{G}}(X)$ equal to $X_{2}$ extended by an element of order 4 .

Lemma 3.4. (1) $G$ has a class $\mathscr{U}$ of subgroups isomorphic to $U_{4}(3)$.

(2) For $U \in \mathscr{U}, U$ has 3 orbits on $\mathscr{U}$ with representatives $U, U_{1}$, and $U_{2}$, where $U \cap U_{1}$ is $L_{3}(4)$ and $U \cap U_{2}$ is $A_{6} / E_{81}$.

(3) $\Delta \cap U$ is the set of long root groups of $U, \Delta \cap U \cap U_{1}=\varnothing$ and $\langle\Delta \cap U \cap$ $\left.U_{2}\right\rangle \in A^{G}$.

As $G$ is of type $M c, H$ has a permutation representation on a set $\Omega=\Omega(t)$ of order 8 with kernel $\langle t\rangle$. Set $H^{*}=H /\langle t\rangle$ and regard $H^{*}$ as $\operatorname{Alt}(\Omega)$. We let $x \leq H$ be a member of $\Delta$; then $x^{*}$ is generated by a 3-cycle.

Lemma 3.5. (1) $x$ is inverted by no involution in $G$.

(2) For each involution $i \in H-\langle t\rangle, i^{*}$ is fixed point free on $\Omega$.

Proof. Part (2) is a property of the covering group of $A_{8}$. If (1) fails then $x$ is inverted by an involution in $H$. But by (2), no involution in $H$ inverts $x$.

Lemma 3.6. Each pair of adjacent vertices in $\Delta$ is contained in a unique conjugate of $A$ and each triangle in $\Delta$ is fused into $A$ under $G$. 
Proof. By Lemma 3.2(6), each edge in $\Delta$ is conjugate to $(x, y)$ for some fixed $y \in \Delta \cap A$. By Lemma 3.2(5), $y \nless R$, so as $|T: R|=3, T$ is the unique Sylow 3-subgroup of $G_{x}$ containing $x y$, and hence $A$ is the unique member of $A^{G}$ containing $x y$. As $\Delta$ is the commuting graph, each triangle of $\Delta$ generates a 3-group, so as $A=\langle\Delta \cap T\rangle$, each triangle of $G$ is fused into $A$.

Lemma 3.7. $N_{G}(X)$ is 2-transitive on $N_{\Delta}(X)$ of order 25 with kernel $X$ and for each pair $x, y \in N_{\Delta}(X), O\left(N_{G}(X)\right)=\langle x, y\rangle$ and there exists an involution in $\widehat{G}$ inverting $X, x$, and $y$.

Proof. Let $Y=N_{G}(X)$ and $\tilde{Y}=Y / X$. Let $y \in \Delta_{4}(x)$ as in Lemma 3.2(6). By Lemma 3.3, $Z(\langle x, y\rangle) \in X^{G}$ so without loss $X=Z(\langle x, y\rangle)$. Indeed by Lemma 3.3, $O(Y)=x P$ with $x \in \operatorname{Syl}_{3}(x P)$, so $P$ is transitive on $N_{\Delta}(X)$, and hence $\left|N_{\Delta}(X)\right|=\left|P: P_{x}\right|=|P: X|=25$. Also $Y=P Y_{x}$ with $\widetilde{Y}_{x}$ a complement to $\widetilde{P}$, so the action of $\tilde{Y}_{x}$ on $N_{\Delta}(X)$ is equivalent to its action on $\widetilde{P}$ by conjugation. By Lemma $3.3, \widetilde{Y}_{x} \cong \mathrm{Z}_{8} / \mathbf{Z}_{3}$ is faithful on $\widetilde{P}$ and hence regular on $\widetilde{P}^{\sharp}$, so $Y$ is 2-transitive on $N_{\Delta}(X)$. Finally by Lemma $3.3(5)$ there exists an involution inverting $X$ and 5 members of $N_{\Delta}(X)$, so the proof is complete.

Lemma 3.8. For $s \in G$ an involution, $x^{s} \notin \Delta_{3}(x)$.

Proof. If so there is an involution in $L=\left\langle x, x^{s}\right\rangle$ that we may take to be $t$ and hence $s \in H$. Now $L^{*} \cong A_{5}$ is the stabilizer of 3 points of $\Omega$ so as $s$ acts on $L$ it must fix one of these 3 points. This contradicts Lemma 3.5(2).

Lemma 3.9. (1) $\operatorname{Fix}_{\mathscr{U}}(t)$ is of order 35 .

(2) For $U \in \operatorname{Fix}_{\mathscr{U}}(t),(H \cap U)^{*}$ is the stabilizer of a partition of $\Omega$ into two halves.

(3) Each dihedral subgroup of $G$ fixes a point of $\mathscr{U}$.

(4) For $y \in \Delta,\langle x, y\rangle$ fixes a point of $\mathscr{U}$ if and only if $y \in x^{\perp} \cup \Delta_{2}(x)$.

(5) If $y \in \Delta$ then $\langle t, y\rangle$ fixes a point of $\mathscr{U}$ if and only if $y^{t} \in y^{\perp} \cup \Delta_{2}(y)$.

(6) If $y \in \Delta$ with $y^{t} \notin \Delta_{4}(y)$ and $K \leq H$ with $K^{*} \cong A_{6}$, then there exists $v \in K \cap\left(y^{\perp} \cup \Delta_{2}(y)\right)$.

(7) If $s$ is an involution in $G$ and $K \leq H$ with $K^{*} \cong A_{6}$, then there exists $v \in \Delta \cap K$ with $v^{s} \in v^{\perp} \cup \Delta_{2}(v)$. Further if $K_{1} \leq C_{G}(s)$ with $K_{1} /\langle s\rangle \cong A_{6}$, then there is $u \in \Delta \cap K_{1}$ with $u \in v^{\perp} \cup \Delta_{2}(v)$.

Proof. Let $t \in U$. Then $U$ has one class of involutions and $C_{U}(t) \cong E_{4} / \mathrm{SL}_{2}(3)$ * $\mathrm{SL}_{2}(3)$, so $H$ is transitive on $\operatorname{Fix}(t)=\operatorname{Fix}_{\mathscr{U}}(t)$ of order $\left|H: C_{U}(t)\right|=35$. Further the unique subgroup of $H$ of index 35 is the stabilizer in (2), so (1) and (2) are established.

Let $D$ be a dihedral subgroup of order $2 m$. If $m$ is even we may take $t \in Z(D)$. But then using Lemma 3.5(2) and (2) we check $D$ fixes a point of $\mathscr{U}$. So $m$ is odd and hence by Lemma $3.1(6), m=3$ or 5 .

If $m=3$ then by Lemmas 3.5(1) and 3.2,O(D) is determined up to conjugation in $G$. Further using Lemma 3.5(2), $H$ is transitive on its $S_{3}$-subgroups 
and on $O(D)^{G} \cap H$, so $G$ is transitive on its $S_{3}$-subgroups. Similarly by Lemma 3.3, $G$ has two classes of elements of order $5, X$ is not inverted by an involution of $G$, and $N_{G}\left(X_{2}\right)$ is transitive on the involutions in $G$ inverting $X_{2}$, so $D$ is determined up to conjugacy in $G$. Finally $U$ has dihedral subgroups of order 6 and 10, so (3) holds.

As the members of $\Delta$ are long root elements of $U_{4}(3)$, if $x \in U$ then $y \in x^{\perp} \cup \Delta_{2}(x)$ for all $y \in U$, and $U$ contains elements in $\Delta_{i}(x)$ for $i \leq 2$. Thus (4) holds. Moreover (4) implies half of (5). Further by Lemma 3.4(3), if $y \in \Delta_{2}(x)$ then $\langle x, y\rangle$ fixes a unique point of $\mathscr{U}$, so if $y^{t} \in \Delta_{2}(y)$ then $t$ fixes the unique point fixed by $\left\langle y, y^{t}\right\rangle$. If $y^{t} \in y^{\perp}$ then $\langle y, t\rangle$ centralizes an involution $s \in G$, and we check that $\langle y, t\rangle$ fixes a partition of $\Omega(s)$ into two halves and hence a member of $\mathscr{U}$. So $(5)$ holds.

Let $K \leq H$ with $K^{*} \cong A_{6}$. Then $K$ is the stabilizer of 2 points of $\Omega$, which allows us to check that each partition of $\Omega$ into two halves is fixed by some member of $\Delta \cap K$. Hence by (2), each member of $\mathrm{Fix}_{\mathscr{U}}(t)$ is fixed by some member of $\Delta \cap K$. This observation together with (5) and Lemma 3.8 implies (6). Similarly under the hypotheses of (7), (3) says $\langle s, t\rangle$ fixes some $U \in \mathscr{U}$ and by the observation there is $v \in \Delta \cap K, u \in \Delta \cap K_{1}$ fixing $U$. Then $u, v$ work in (7) by (4).

In the remainder of this section let $\theta$ be the graph on $\Delta$ with $x$ adjacent to $y$ if and only if $y \in \Delta_{4}(x)$. We wish to show

Theorem 3.10. If $y \in \Delta_{4}(x)$ then $\Delta \cap\langle x, y\rangle$ is a maximal clique in $\theta$

The proof involves a number of lemmas. Let $y \in \Delta_{4}(x)$; we may assume $X=Z(\langle x, y\rangle)$. Set $Y=N_{G}(X)$ and $\widehat{Y}=N_{\widehat{G}}(X)$. We must show the set $\theta(\Delta \cap Y)$ of vertices adjacent in $\theta$ to each member of $\Delta \cap Y$ is equal to $\Delta \cap Y$. By Lemma 3.7, $\Delta \cap Y \subseteq \theta(\Delta \cap Y)$, so it remains to show that if $z \in \theta(\Delta \cap Y)$ then $z \leq Y$.

Lemma 3.11. Let $a$ and $b$ be distinct elements of $\Delta$. Then

(1) If $\langle a, b\rangle \leq A$ then $\operatorname{Fix}_{\mathscr{U}}(\langle a, b\rangle)=\operatorname{Fix}_{\mathscr{U}}(A)$ is of order 2 .

(2) If $\langle a, b\rangle \leq U \in \mathscr{U}$ then $\Delta(a, b) \subseteq U$.

(3) $|\Delta(a, b)| \leq 8$.

(4) $\left|\operatorname{Fix}_{\mathscr{U}}(a)\right|=5$ and $\operatorname{Fix}_{\mathscr{U}}(a)$ and $\operatorname{Mov}_{\mathscr{U}}(a)$ are orbits of $G_{a}$, with $\mid \Delta(a) \cap$ $V \mid=1$ for $V \in \operatorname{Mov}_{\mathscr{U}}(a)$.

Proof. Let $U \in \mathscr{U}$. As $U$ is transitive on edges of $\Delta$ in $U, G_{a, b}$ is transitive on $\operatorname{Fix}_{\mathscr{U}}(\langle a, b\rangle)$ if $\langle a, b\rangle \leq A$. Similarly $N_{G}(A)$ is transitive on $\operatorname{Fix}_{\mathscr{U}}(A)$ and $G_{a}$ is transitive on $\operatorname{Fix}_{\mathscr{U}}(a)$.

Next $G_{a, b} \leq N_{G}(A)$ by Lemma 3.6, so $\operatorname{Fix}_{\mathscr{U}}(\langle a, b\rangle)=\operatorname{Fix}_{\mathscr{U}}(A)$ and by Lemmas 3.4(3) and 3.2(3), $\left|N_{G}(A): N_{U}(A)\right|=2$ for $U \in \operatorname{Fix}_{\mathscr{U}}(A)$. So (1) holds. Similarly $\left|\operatorname{Fix}_{\mathscr{U}}(a)\right|=\left|G_{a}: U_{a}\right|=5$. Also by Lemma 3.6, $\Delta(a, b) \subseteq A$ so (2) and (3) hold in this case.

If $\langle a, b\rangle \leq U$ but $b \notin \Delta(a)$, then by Lemma 3.4(3), $\{U\}=\operatorname{Fix}_{\mathscr{U}}(\langle a, b\rangle)$, so (2) holds in this case too, and implies (3). If $\operatorname{Fix}_{\mathscr{U}}(\langle a, b\rangle)=\varnothing$ then $\Delta(a, b) \subseteq C_{G}(Z(\langle a, b\rangle))$, and we check $|\Delta(a, b)| \leq 1$ inside the later group. So (3) is established. 
We have seen $\operatorname{Fix}_{\mathscr{U}}(a)$ is an orbit of $G_{a}$ of length 5 . Further by (1), each $b \in$ $\Delta(a)$ fixes exactly 3 points of $\operatorname{Mov}_{\mathscr{U}}(a)$, while by $(2), \operatorname{Mov}_{\mathscr{U}}(a) \cap \operatorname{Fix}(\langle b, c\rangle)=$ $\varnothing$ for distinct $b, c \in \Delta(a)$. Thus there are $3 \cdot|\Delta(a)|=270$ members of $\operatorname{Mov}_{\mathscr{U}}(a)$ containing a member of $\Delta(a)$ and that member is unique. Finally $|\mathscr{U}|=275, G_{a}$ is transitive on $\Delta(a)$, and $a$ is transitive on $\operatorname{Fix}_{\mathscr{U}}(b)-\operatorname{Fix}(a b)$, so (4) is established.

Lemma 3.12. (1) $Y$ has two orbits $\mathscr{U}_{1}$ and $\mathscr{U}_{2}$ on $\mathscr{U}$ of lengths 125 and 150, respectively.

(2) $U \cap Y \cap \Delta \neq \varnothing$ if and only if $U \in \mathscr{U}_{1}$, in which case $\widehat{Y} \cap \widehat{U}$ is a complement to $P$ in $\widehat{Y}$.

Proof. By Lemma 3.11(4), $X$ is regular on $\operatorname{Fix}_{\mathscr{U}}(x)$, so for $U \in \operatorname{Fix}_{\mathscr{U}}(x)$, $U Y$ is of order 125 with $\widehat{Y} \cap \widehat{U}$ a complement to $P$ in $\widehat{Y}$ and consists of those members of $\mathscr{U}$ fixed by some member of $\Delta \cap Y$. Similarly $X_{2}$ fixes some $V \in \mathscr{U}$ and $N_{V}\left(X_{2}\right)=Y \cap V$ is a Frobenius group of order 20, so $|V Y|=|Y: Y \cap V|=150$, and hence $V Y=\mathscr{U}-U Y$.

In the remainder of this section let $\Lambda=\theta(Y \cap \Delta)-(Y \cap \Delta)$ and $b \in \Lambda$.

Lemma 3.13. $\widehat{Y}_{b}=1$.

Proof. Suppose $g \in \widehat{Y}_{b}$ is of prime order $p$ and let $C=C_{G}(g)$. If $p=5$ then $g \notin X$ as $b \notin Y$, and then Lemma 3.3 contradicts $b \leq C$. If $p=3$ then $\langle g\rangle \in \Delta(b) \cap Y$, contradicting $b \in \Lambda$. So $p=2$. If $g \in G$ then $g$ centralizes some $x \in \Delta \cap Y$ while by Lemma $3.5(1), b \leq C$, so $\langle x, b\rangle \leq C$, contradicting $b \in \Delta_{4}(x)$.

Thus $g \in \widehat{Y}-Y$ is an involution. Then $g$ inverts 5 members $y_{i}, 1 \leq i \leq 5$, of $Y \cap \Delta$ and $b$. By Lemma 3.1(3), $C \cong M_{11}$ acts faithfully on a set $\Xi$ of order 11, and by Lemma 3.2(7), $C_{y_{i}} \cong M_{9}$ is the stabilizer in $C$ of some 2-subset $\xi(i)$ of $\Xi$ and similarly $C_{b}=C_{\xi(b)}$. If $C_{y_{i}} \cap C_{y_{j}} \neq 1$ then there is some $d$ of order 2 or 3 in $C_{C}\left(\left\langle y_{i}, y_{j}\right\rangle\right)$, contradicting $y_{j} \in \Delta_{4}\left(y_{i}\right)$. So $\xi(i) \cap \xi(j)=\varnothing$, and similarly $\xi(i) \cap \xi(b)=\varnothing$. But then the six 2-subsets $\xi(i), \xi(b), 1 \leq i \leq 5$, are pairwise disjoint, contradicting $|\Xi|=11$.

Lemma 3.14. Let $b \leq U \in \mathscr{U}, \Lambda(U)=\Lambda \cap U$, and $\varepsilon(U)=U \cap \Delta-\Lambda(U)$. Then

(1) $U \in \mathscr{U}_{2}$ in the notation of Lemma 3.12 .

(2) $|\Lambda(U)| \geq 200$ and $|\varepsilon(U)| \leq 80$.

Proof. Part (1) follows from Lemmas 3.12(2) and 3.9(4). For the first inequality in (2), count the set $\mathscr{T}$ of pairs $(a, V)$ with $a \in b^{Y}, V \in \mathscr{U}$, and $a \leq V$ in two ways. By Lemma 3.13 there are $|\widehat{Y}|=6,000$ choices for $a \in b^{\widehat{Y}}$ and by (1) and Lemma 3.12(1) there are 150 choices for $V \in \mathscr{U}_{2}$ and $a$ is contained in 5 members of $\mathscr{U}_{2}$, so $U$ contains $200 \widehat{Y}$-conjugates of $b$. Finally as $|U \cap \Delta|=280$, the first inequality implies the second. 
We now complete the proof of Theorem 3.10 by proving $|\varepsilon(U)|>80$, contradicting Lemma 3.14. Define

$$
\mathscr{A}=\bigcup_{y \in Y \cap \Delta} \Delta(y) \cap U .
$$

For $u \in \mathscr{A},|\Delta(u) \cap Y|=1$ and $\Delta(u) \cap U \subseteq \varepsilon(U)$ as $z \notin \Delta_{4}(x)$ for $x \in \Delta(u) \cap Y$ and $z \in \Delta(u)$. Further by Lemmas 3.11(4) and 3.14(1), $|\Delta(x) \cap U|=1$, so $|\mathscr{A}|=25$. However for each $u \in \mathscr{A},|\Delta(u) \cap U|=36$ so by Lemma 3.11(3),

$$
|(\Delta(u) \cap U) \cup(\Delta(v) \cup U) \cup(\Delta(w) \cap U)| \geq 3 \cdot 36-3 \cdot 8=84,
$$

for distinct $u, v, w \in \mathscr{A}$. Thus the proof of Theorem 3.10 is at last complete.

\section{SOME LOCAL SUBGROUPS OF GROUPS OF TYPE $L y$}

In this section we assume $G$ is a group of type $L y$. Thus $t \in G$ is an involution, $H=C_{G}(t), t$ is not weakly closed in $H$ with respect to $G$ and $H$ is the covering group of $A_{11}$. Thus we have a permutation representation of $H$ on a set $\Omega=\Omega(t)$ of order 11 with kernel $\langle t\rangle$. Set $H^{*}=H /\langle t\rangle$, and regard $H^{*}$ as $\operatorname{Alt}(\Omega)$. For $h \in H$, let $\operatorname{Fix}_{\Omega}(h)$ and $\operatorname{Mov}(h)$ denote the members of $\Omega$ fixed and moved by $h$, respectively.

We take $\Omega=\{1, \ldots, 11\}$ and let $x_{i}, 1 \leq i \leq 3$, be elements of order 3 in $H$ such that

$$
x_{1}^{*}=(1,2,3), \quad x_{2}^{*}=(4,5,6), \quad x_{3}^{*}=(7,8,9) .
$$

Let $\Delta$ be the commuting graph on $\left\langle x_{1}\right\rangle^{G}$; we also write $\Delta$ for the vertices of this graph.

Lemma 4.1. (1) $H$ is transitive on involutions in $H-\langle t\rangle$.

(2) If $s \in H-\langle t\rangle$ is an involution then $s^{*}$ has 4 cycles of length 2 on $\Omega$.

(3) $G$ has one conjugacy class of involutions.

(4) $G$ is simple.

Proof. Parts (1) and (2) are well-known properties of the covering group $H$ of $A_{11}$. Now by hypothesis $t$ is not weakly closed in $H$ with respect to $G$, so (1) implies (3). Then Lemma 2.1 implies (4).

Lemma 4.2. $x_{1}$ is not conjugate to $x_{1} x_{2}$ or $x_{1} x_{2} x_{3}$ in $G$.

Proof. Let $y=x_{1} x_{2}$ and $T, S$ be Sylow 2-subgroups of $C_{H}\left(x_{1}\right), C_{H}(y)$, respectively. Then $\langle t\rangle=Z(T)=Z(S)$ and $S$ is semidihedral of order 16. As $\langle t\rangle$ is the center of $T, T \in \operatorname{Syl}_{2}\left(C_{G}\left(x_{1}\right)\right)$ and similarly $S \in \operatorname{Syl}_{2}\left(C_{G}(y)\right)$. Thus as $|T| \neq|S|, x$ is not conjugate to $y$.

Similarly let $u=y x_{3}$. Then a Sylow 2-subgroup of $C_{H}(u)$ is a 4-group, so by a lemma of Suzuki (cf. Exercise 8.6 in [1]), a Sylow 2-subgroup of $C_{G}(u)$ is dihedral or semidihedral, so again $u$ and $x_{1}$ are not conjugate.

Lemma 4.3. $C_{G}\left(x_{1}\right) /\left\langle x_{1}\right\rangle \cong M c$ and $N_{G}\left(\left\langle x_{1}\right\rangle\right) /\left\langle x_{1}\right\rangle \cong \operatorname{Aut}(M c)$.

Proof. Let $M=C_{G}\left(x_{1}\right), \widehat{M}=N_{G}\left(\left\langle x_{1}\right\rangle\right)$, and $\widehat{M}^{*}=\widehat{M} /\left\langle x_{1}\right\rangle$. First $C_{M}(t)=$ $C_{H}\left(x_{1}\right)=\left\langle x_{1}\right\rangle \times L$, where $L$ is the covering group of $A_{8}$. Further by Lemma 
4.2, $x_{1}^{G} \cap H=x_{1}^{H}$, so $M$ has one class of involutions. Thus $M^{*} \cong M c$ by Lemma 3.1. As an element $h$ of $H$ inverting $x_{1}$ induces an outer automorphism on $L, h$ induces an outer automorphism on $M^{*}$; then as $|\operatorname{Out}(M c)|=$ $2, \widehat{M}^{*} \cong \operatorname{Aut}(M c)$.

Continue the notation of the proof of Lemma 4.3 for a few more moments, and let $A^{*}$ be the weak closure of $x_{2}^{*}$ in a Sylow 3-subgroup $T^{*}$ of $M^{*}$. By Lemmas 4.3 and 3.2(3), $A^{*} \cong E_{81}, N_{\widehat{M}^{*}}\left(A^{*}\right)$ is the split extension of $A^{*}$ by $\mathbf{Z}_{2} \times M_{10}$ with $\Delta_{A}=\left\langle x_{2}\right\rangle^{N_{M}(A)} \cup\left\{\left\langle x_{1}\right\rangle\right\}$ of order 11 and some involution $s \in \widehat{M}-M$ inverts $A^{*}$.

Then $s$ inverts $A$, so $A \cong E_{3^{5}}$. Each element of $M^{*}$ of order 3 is fused to $x_{2}^{*}$ or $x_{2}^{*} x_{3}^{*}$, so by Lemma $4.2, \Delta_{A}=\Delta \cap T$. As $\left|\Delta_{A}\right|=11$ is prime to 3 , a Sylow 3-subgroup of $N_{G}(T)$ fixes some member of $\Delta_{A}$, and hence $T \in \operatorname{Syl}_{3}(G)$ and $N_{G}(A)$ controls fusion in $A$. In particular, $N_{G}(A)$ is transitive on $\Delta_{A}$, so as $N_{\widehat{M}}(A) /\langle s\rangle A$ is sharply 3 -transitive on $\Delta_{A}-\left\{\left\langle x_{1}\right\rangle\right\}, N_{G}(A) /\langle s\rangle A$ is sharply 4transitive on $\Delta_{A}$. It follows that $N_{G}(A)$ is the split extension of $A$ by $\mathbf{Z}_{2} \times M_{11}$. We have shown

Lemma 4.4. Let $T \in \operatorname{Syl}_{3}(G)$ and $A=\langle\Delta \cap T\rangle$. Then $A \cong E_{3^{5}},|\Delta \cap T|=11$, and $N_{G}(A)$ is the split extension of $A$ by $\mathbf{Z}_{2} \times M_{11}$.

Lemma 4.5. (1) $T=R A$ where $R \cong 3^{2+4}$.

(2) All elements of order 3 in $T$ are contained in $R$ or $A$.

(3) $\left\{\left\langle x_{1}\right\rangle,\left\langle x_{2}\right\rangle\right\}=\Delta \cap R$.

(4) $G$ has two classes of elements of order 3 with representatives $x_{1}$ and $x_{1} x_{2}$.

(5) $C_{G}\left(x_{1}\right)$ is quasi-simple and the covering group of $M c$.

Proof. Let $M^{*}=M /\left\langle x_{1}\right\rangle$. By Lemmas 4.3 and 3.1(4), $T^{*}=R^{*} A^{*}$ with $R^{*} \cong$ $3^{1+4}$, all elements of order 3 in $T^{*}$ contained in $R^{*}$ or $A^{*}$, and $\left\langle x_{2}^{*}\right\rangle^{M} \cap R=$ $\left\langle x_{2}^{*}\right\rangle$. Therefore (2) is established.

By (2), $R$ is characteristic in $T$. Further $\left\langle x_{2}^{*}\right\rangle=Z\left(R^{*}\right)$ and $x_{2}$ is inverted in $N_{M}(R)$, so $x_{2} \in \Phi(R)$. Then as $\left\langle x_{1}\right\rangle$ is conjugate to $\left\langle x_{2}\right\rangle$ in $N_{G}(T)$, $\left\langle x_{1}, x_{2}\right\rangle=\Phi(R)$. Therefore $R \cong 3^{2+4}$ and (1) holds. Also by Lemma 4.3, $C_{G}\left(x_{1}\right)$ is quasi-simple and hence (5) holds by Lemma 3.1(4). As each element of order 3 in $M$ is fused under $M$ into $A$ and $M_{11}$ has two orbits on $A^{\sharp}$, parts (3) and (4) hold by Lemma 4.2.

Lemma 4.6. (1) $N_{G}\left(\left\langle x_{1}, x_{2}\right\rangle\right)$ is of index 2 in $N_{G}\left(\left\langle x_{1}, x_{2}\right\rangle\right)$.

(2) $N_{G}\left(\left\langle x_{1}, x_{2}\right\rangle\right)$ is the split extension of $R$ by a complement to $\left\langle x_{1}, x_{2}\right\rangle$ in $N_{H}\left(\left\langle x_{1}, x_{2}\right\rangle\right)$, with $t$ inverting $R /\left\langle x_{1}, x_{2}\right\rangle$.

Proof. Let $y=x_{1} x_{2}, E=\left\langle x_{1}, x_{2}\right\rangle, N=N_{G}(E)$, and $D=C_{G}(y)$. By Lemmas 4.2 and 4.5(4), $H$ has two orbits on $y^{G} \cap H$, so $D$ has two classes of involutions. However we saw during the proof of Lemma 4.2 that $S \in$ $\operatorname{Syl}_{2}\left(C_{D}(t)\right)$ is semi-dihedral and Sylow in $D$, so by Thompson transfer $D$, has a subgroup of index 2 with quaternion Sylow 2-subgroups, and then by a 
result of Brauer and Suzuki [3], $D=O(D) C_{D}(t)$. Now $E=O\left(C_{D}(t)\right) \leq O(D)$. Conversely if $s$ is an involution in $C_{D}(t)-\langle t\rangle$ then $C_{O(D)}(s) \leq O\left(C_{G}(\langle s, y\rangle)\right)$, which is a 3-group, so $O(D)$ is a 3-group. Then $\langle\Delta \cap O(D)\rangle \leq T$, while $R=$ $O\left(C_{M}\left(x_{2}\right)\right)$, so $O(D) \leq R$. Then by Lemma 4.5(3), $\left\{\left\langle x_{1}\right\rangle,\left\langle x_{2}\right\rangle\right\}=\langle\Delta \cap O(D)\rangle \unlhd$ $D$, so (1) holds and $|N: N \cap M|=2$ and hence indeed $R=O(D)$. Similarly as $N \cap M=R\left(C_{N \cap M}(t)\right), N=R C_{N}(t)$. Hence as $N \cap H$ splits over $E$, the proof is complete.

Lemma 4.7. Let $X \leq H$ be of order 5 with $X^{*}=\langle(7,8,9,10,11)\rangle$. Then $N_{G}(X)$ is the split extension of $P=O_{5}\left(N_{G}(X)\right)$ by a complement to $X$ in $N_{H}(X)$ and $P \cong 5^{1+4}$.

Proof. Let $L=C_{H}(X), Y=C_{G}(X)$, and $P=O(Y)$. Then $L \cong \mathrm{SL}_{2}(9)$ and $E=\left\langle x_{1}, x_{2}\right\rangle \in \operatorname{Syl}_{3}(L)$. By Brauer-Suzuki [3], $Y=P C_{Y}(t)$. Now from Lemmas 4.3 and $3.3(2), O\left(C_{G}\left(X\left\langle x_{i}\right\rangle\right)\right)=E P_{i}$ with $P_{i} \cong 5^{1+2}$, so in particular $C_{P}\left(x_{i}\right)=P_{i}$ as $E \cap O\left(C_{Y}(t)\right)=1$ and $t$ inverts $P_{i} / X$. Similarly by Lemma 4.6, $O\left(C_{G}\left(\left\langle x_{1} x_{2}\right\rangle X\right)\right)=X E$, so $C_{P}\left(x_{1} x_{2}\right)=X$. It follows that $P$ is the central product of $P_{1}$ and $P_{2}$ and hence $P \cong 5^{1+4}$. The lemma follows.

Lemma 4.8. Let $X_{2} \leq H$ be of order 5 with $X_{2}^{*}=\langle(2,3,4,5,6)(7,8,9,10$, 11)). Then $X_{2} \leq O_{5}\left(N_{G}\left(X_{1}\right)\right)$ for some $X_{1} \in X^{G}$ and $N_{G}\left(X_{2}\right) \leq N_{G}\left(X_{1}\right)$. Further $C_{G}\left(X_{2}\right)$ is the extension of $\mathbf{Z}_{5} \times 5^{1+2}$ by $S_{3}$ with a Sylow 3-subgroup of $C_{G}\left(X_{2}\right)$ in $\Delta$.

Proof. Continue the notation of Lemma 4.7. There exists an involution $s \in H$ inverting $X$ and $\left\langle x_{1}\right\rangle$ and as $t$ inverts $C_{P}\left(x_{1}\right) / X, C_{P}\left(\left\langle x_{1}, r\right\rangle\right) \neq 1$ for $r=s$ or $s t$, say $r=s$. Let $U$ be of order 5 in $C_{P}\left(\left\langle x_{1}, s\right\rangle\right)$.

Now by Lemma 3.3(4), $X$ is weakly closed in $N_{M}(X)$ with respect to $M$ and by Lemmas 4.2 and 4.7, $\Delta \cap Y=\left\langle x_{1}\right\rangle^{Y}$, so $X$ is also weakly closed in $N_{M}(X)$ with respect to $G$. In particular $U \notin X^{G}$. Hence as $H$ has two classes of subgroups of order 5 with representatives $X$ and $X_{2}$, we conclude $U \in X_{2}^{G}$.

Next $C_{H}\left(X_{2}\right)=X X_{2}\langle t\rangle$, so $\langle t\rangle \in \operatorname{Syl}_{2}\left(C_{H}\left(X_{2}\right)\right)$ and thus $\langle t\rangle \in \operatorname{Syl}_{2}\left(C_{G}\left(X_{2}\right)\right)$. Therefore $\langle s\rangle \in \operatorname{Syl}_{2}\left(C_{G}(U)\right)$, so by Thompson transfer $C_{G}(U)=O\left(C_{G}(U)\right)\langle s\rangle$. Also as $C_{H}(t)=X X_{2}\langle t\rangle, \quad C_{O\left(C_{G}(U)\right)}(s)=C_{P}(s)$. By Lemma 3.3(3), $C_{P}\left(\left\langle x_{1}, U\right\rangle\right)\left\langle x_{1}\right\rangle=C_{M}(U)$, so $\left\langle x_{1}\right\rangle \in \operatorname{Syl}_{3}\left(C_{G}(U)\right)$ and hence $O\left(C_{G}(U)\right)=$ $O_{\{2,3\}^{\prime}}\left(C_{G}(U)\right)\left\langle x_{1}\right\rangle$ and $C_{Q}\left(x_{1}\right)=1=C_{Q}(s)$ for each $\left\langle x_{1}, s\right\rangle$-invariant Sylow $p$-subgroup of $O_{\{2,3\}^{\prime}}\left(C_{G}(U)\right)$ and each prime $p \neq 5$. As $\left\langle x_{1}, s\right\rangle \cong S_{3}$ it follows that $O_{\{2,3\}^{\prime}}\left(C_{G}(U)\right)$ is a 5-group.

Finally $h_{Y}\left(x_{1}, 5\right)=\{P\}$, so $C_{P}(U)=O_{5}\left(N_{G}(U)\right) \cap N_{G}(X)$. Also $C_{P}(U) \cong$ $\mathrm{Z}_{5} \times 5^{1+2}$ with $X=\Phi\left(C_{P}(U)\right)$, so $C_{P}(U)=O_{5}\left(N_{G}(U)\right)$. Thus the lemma is established.

\section{THE LYONS GRAPH AND THE GROUP ORDER}

In this section we continue the hypotheses and notation of $\S 4$. In particular recall $\Delta$ is the commuting graph on the conjugates of $\left\langle x_{1}\right\rangle$. We often write 
$x$ for a member of $\Delta$. Note this means $x$ is a subgroup of order 3 , not an element of order 3 .

For $x \in \Delta$, we define three subsets $\Delta_{i}^{2}(x)$ of $\Delta$ as follows: $\Delta_{i}^{2}(x)$ consists of all $y \in \Delta$ such that $\langle x, y\rangle \cong \mathrm{SL}_{2}(3), \mathrm{SL}_{2}(5), \mathrm{Z}_{3} / 5^{1+2}$, for $i=1,2,3$, respectively. Write $G_{x}$ for $N_{G}(x)$.

Lemma 5.1. For each $x \in \Delta$ :

(1) $\Delta(x)$ and $\Delta_{i}^{2}(x)$ are orbits of $G_{x}$ on $\Delta$.

(2) The map $y \mapsto x y / x$ is a bijection between $\Delta(x)$ and the set of 3-central subgroups of $G_{x} / x$ of order 3 .

(3) $|\Delta(x)|=2^{3} \cdot 5^{2} \cdot 7 \cdot 11=15,400$ and $G_{x, y} \cong \mathbf{Z}_{2} /\left(\mathbf{Z}_{4} * \mathrm{SL}_{2}(5)\right) / 3^{2+4}$.

(4) $\left|\Delta_{1}^{2}(x)\right|=2^{3} \cdot 3^{5} \cdot 5^{2} \cdot 11=534,600$ and $G_{x, y} \cong S_{7} / Z_{2}$.

(5) $\left|\Delta_{2}^{2}(x)\right|=2^{2} \cdot 3^{5} \cdot 5^{2} \cdot 7 \cdot 11=1,871,100$ and $G_{x, y} \cong S_{6} / \mathbf{Z}_{2}$.

(6) $\left|\Delta_{3}^{2}(x)\right|=2^{7} \cdot 3^{6} \cdot 7 \cdot 11=7,185,024, G_{x, y} \cong S_{3} / 5^{1+2}$, and for each pair of distinct $u, v \in \Delta(x, y), v \in \Delta_{3}^{2}(u)$ with $\Delta(x, y)$ consisting of the 25 members of $\langle u, v\rangle \cap \Delta$.

Proof. Without loss $x=\left\langle x_{1}\right\rangle$. Let $\widehat{M}=G_{x}, M=C_{G}(x)$, and $\widehat{M}^{*}=\widehat{M} / x$. Now $z=\left\langle x_{2}\right\rangle \in \Delta(x)$ and by Lemma 4.5 , (2) holds and $\Delta(x)$ is an orbit of $M$.

Next by Lemma $3.2(2), N_{\widehat{M}^{*}}\left(z^{*}\right)$ is the split extension of $R^{*} \cong 3^{1+4}$ by $\mathrm{Z}_{2} /\left(\mathbf{Z}_{4} * \mathrm{SL}_{2}(5)\right)$. Further by Lemma $4.5, R \cong 3^{2+4}$ and $N_{\widehat{M}^{*}}\left(z^{*}\right)=G_{x, z}^{*}$, so $G_{x, z}$ is as claimed in (3). So (3) is established.

Next if $y \in \Delta_{i}^{2}(x)$ for $i=1$ or 2 , then $\langle x, y\rangle$ contains a unique involution, and as $M$ has one class of involutions, we may choose that involution to be $t$. Then $G_{x, y}=H_{x, y}$, so the structure of $G_{x, y}$ is easily calculated from its action on $\Omega$. By 9-transitivity of $H$ on $\Omega$ and Lemma 4.2, $H_{x}$ is transitive on $\Delta_{i}^{2}(x) \cap H$, so $\Delta_{i}^{2}(x)$ is an orbit of $M$ and (4) and (5) are established.

Finally let $y \in \Delta_{3}^{2}(x)$ and $L=\langle x, y\rangle$. Then $Z(L) \cong Z_{5}$ and as $M$ has two classes of elements of order 5 , we may take $Z(L)=X$ or $X_{2}$, discussed in Lemmas 4.7 and 4.8, respectively. But by Lemma 4.8, $X_{2} \npreceq \Phi(Q) Q \in$ $\operatorname{Syl}_{5}\left(C_{G}\left(X_{2}\right)\right)$, so $Z(L)=X$. By Lemma 4.7, $\Lambda_{C(X)}(x, 5) \subseteq \stackrel{P}{=}=O_{5}\left(C_{G}(X)\right)$, so $O_{5}(L) \leq P$. Now from the proof of Lemma 4.7, $[P, x]=C_{P}\left(x_{2}\right) \cong 5^{1+2}$, so $O_{5}(L)=[P, x]$. Also $K=\left\langle x_{2}\right\rangle\left[P, x_{2}\right] \leq C_{G}(L)$ with $K=\left\langle x_{2}, v\right\rangle$ for any $\left\langle x_{2}\right\rangle \neq v \in x_{2}^{K}$ and $v \in \Delta_{3}^{2}\left(\left\langle x_{2}\right\rangle\right)$. It remains only to observe that $N_{G}(X\langle x\rangle)$ is transitive on the 24 members of $\Delta \cap L-\{x\}$, so that indeed $\Delta_{3}^{2}(x)$ is an orbit of $\widehat{M}$ and that $G_{x, y}=N_{G}(X)_{x, y}=K\langle s\rangle$, where $s$ is an involution inverting $x_{2}$. Hence (6) holds and the proof is complete.

Lemma 5.2. For each $x \in \Delta$,

$$
\Delta^{2}(x)=\Delta_{1}^{2}(x) \cup \Delta_{2}^{2}(x) \cup \Delta_{3}^{2}(x) .
$$

Proof. This is a consequence of Lemmas 5.1(2) and 3.1(6).

Lemma 5.3. Let $Y=C_{G}(t)$ or $N_{G}(X)$. Then for all $x, y \in \Delta \cap Y, d(x, y) \leq 2$. 
Proof. If $Y=C_{G}(t)$ this is Lemma 2.2. So let $Y=N_{G}(X), P=O_{5}(Y)$, and $Y^{*}=Y / P$. We saw during the proof of Lemma 5.1 that if $y^{*}=x^{*}$ then $y \in \Delta_{3}^{2}(x)$, while if $y^{*} \neq x^{*}$ but $\left[x^{*}, y^{*}\right]=1$ then $y \in \Delta(x)$. Further by Lemma 4.7, $O^{3^{\prime}}\left(Y^{*}\right) \cong \mathrm{SL}_{2}(9)$, so if $\left[x^{*}, y^{*}\right] \neq 1$ then $\left\langle x^{*}, y^{*}\right\rangle \cong \mathrm{SL}_{2}(3)$ or $\mathrm{SL}_{2}(5)$. We claim $\langle x, y\rangle \cong \mathrm{SL}_{2}(3)$ or $\mathrm{SL}_{2}(5)$ in the respective case, so that $y \in \Delta^{2}(x)$ and the lemma holds.

The claim follows from an easy counting argument. Namely $\left|P: C_{P}(x)\right|=$ 25 , so there are $5^{4}$ pairs $(u, v)$ with $u, v \in \Delta \cap Y, u^{*}=x^{*}$, and $v^{*}=y^{*}$. But if $L \leq Y$ with $L$ a complement to $P$ in the preimage of $\langle x, y\rangle^{*}$ then $X=N_{P}(L)$, so there are $5^{4}$ conjugates of $L$ in $L P$, and hence these are the $5^{4}$ subgroups $\langle u, v\rangle$.

Lemma 5.4. Let $x \in \Delta$ and $y \in \Delta(x)$. Then

(1) $y$ is not inverted by an involution centralizing $x$.

(2) $G_{x}$ is transitive on involutions $s \in G_{x}-C_{G}(x)$ and $C_{G}(\langle x, s\rangle) \cong M_{11}$.

(3) If $s$ inverts $u \in \Delta$ then $d(x, u) \leq 2$.

Proof. Part (1) follows from Lemmas 3.5 and 5.1(2). Part (2) is Lemma 3.1(3). So assume $u \in \Delta$ is inverted by $s$, and let $L=C_{G}(\langle s, x\rangle)$ and $K=C_{G}(\langle s, u\rangle)$. Without loss $s=t$. Now $L \cong K \cong M_{11}$ by (2), and $\left|M_{11}\right|^{2}>2\left|A_{11}\right|$, so $L \cap K \neq 1$. So let $g \in L \cap K$ be of prime order $p$. Then $x, u \in C_{G}(g)$. If $p=2$ then $d(x, u) \leq 2$ by Lemma 5.3. If $p=3$ then by Lemma 4.5(4), $g$ is conjugate to $x_{1}$ or $x_{1} x_{2}$. In either case Lemma 4.6 says $O^{2}\left(C_{G}(g)\right) \leq G_{v}$ for some $v \in \Delta$ so $v \in x^{\perp} \cap u^{\perp}$ and clearly $d(x, u) \leq 2$.

If $p=11$ then $N_{H^{*}}\left(\left\langle g^{*}\right\rangle\right) \leq L^{*}$, so $L^{*}$ is the unique conjugate of $L^{*}$ containing $g^{*}$, and hence $L=K$ and $x=u$. This leaves the case $p=5$. Then as $g \in L,\langle g\rangle$ is conjugate to $X_{2}$. Hence by Lemma 4.8, $C_{G}(g)$ is contained in some conjugate of $N_{G}(X)$, so Lemma 5.3 completes the proof.

Lemma 5.5. If $x, y \in \Delta$ with $\left|G_{x, y}\right|$ even then $d(x, y) \leq 2$.

Proof. Let $t$ be an involution in $G_{x, y}$. By Lemmas 5.3 and 5.4 we may assume $x \in H$ but $t$ inverts $y$. Thus we may take $x=\left\langle x_{1}\right\rangle$ and by Lemma 5.4, $L=C_{G}(\langle y, t\rangle) \cong M_{11}$. Now each element $a$ of order 3 in $L$ has three 3 -cycles on $\Omega$ and by 4-transitivity of $L$ on $\Omega$, we may assume $x_{1}$ is one of those 3-cycles. Thus $x, y \in C_{G}(a)$, and hence as in the proof of the previous lemma, $d(x, y) \leq 2$.

Lemma 5.6. Let $y \in \Delta, u \in \Delta(y), x \in \Delta_{i}^{2}(y)$ for $i=1$ or 2 , and $t$ the involution in $\langle x, y\rangle$. Then

(1) $u^{t} \notin \Delta_{2}^{2}(u)$.

(2) If $u^{t} \in u^{\perp} \cup \Delta_{1}^{2}(u)$ then there exists $v \in \Delta(x, y)$ with $v \in u^{\perp} \cup \Delta_{1}^{2}(u)$.

Proof. Part (1) follows from Lemma 3.8. Similarly as $x \in \Delta_{i}^{2}(y)$ for $i=1$ or 2, $H_{y}$ contains a subgroup $K$ with $K^{*} \cong A_{6}$, so Lemma 3.9(6) implies (2). 
Lemma 5.7. If $p=x e y u$ is a path in $\Delta$ with $y \in \Delta_{1}^{2}(x)$ and $i$ is the involution in $\langle x, y\rangle$ then one of the following holds:

(1) $\left|G_{x, y, u}\right|$ is even.

(2) $x^{\perp} \cap y^{\perp} \cap u^{\perp} \neq \varnothing$.

(3) $u \in \Delta_{2}^{2}(x)$ and $u^{i} \in \Delta_{3}^{2}(u)$.

Proof. Without loss $x=\left\langle x_{1}\right\rangle$ and $t$ is the involution in $\langle x, y\rangle$. Assume neither (1) nor (2) holds. Then as (1) fails, $u \neq u^{t}$.

Suppose $u^{t} \in \Delta(u)$. Then $B=\left\langle y, u, u^{t}\right\rangle \leq T \in \operatorname{Syl}_{3}(G)$ and thus $B \leq$ $A=\langle\Delta \cap T\rangle$. Then $N_{G}(A)$ is described in Lemma 4.5 and by Lemma 3.6, $A$ is the unique conjugate of $A$ containing $B$, so $t \in N_{G}(A)$. As $[y, t]=$ $1, C_{A}(t) \cong E_{27}$ contains exactly 3 members $y, v, w$ of $\Delta$. As $y \in \Delta_{1}^{2}(x)$, $|\operatorname{Mov}(x) \cap \operatorname{Mov}(y)|=2$, so $\operatorname{Mov}(x) \cap \operatorname{Mov}(z)=\varnothing$ for $z=v$ or $w$, and hence $z \in \Delta(x, y, u)$. But then (2) holds.

Assume next that $u^{t} \in \Delta_{1}^{2}(u)$. Let $r$ be the involution in $\left\langle u, u^{t}\right\rangle$ and notice $r \in H$ and $\operatorname{Fix}_{\Omega}(r)=\operatorname{Mov}(y)$. Thus $|\operatorname{Mov}(x) \cap \operatorname{Fix}(r)|=2$. Hence as $x \neq y$, $x^{r} \in \Delta_{1}^{2}(x)$.

Let $L=O^{2}\left(C_{G}(\langle t, r\rangle)\right)$. Then $L^{*}=y^{*} \times L_{0}^{*}$ with $L_{0}^{*} \cong \mathbf{Z}_{3} / 2^{1+4}$ the centralizer of $r^{*}$ in $\operatorname{Alt}(\operatorname{Mov}(r))$. Let $K=L_{x}$; then $K \cong \mathrm{SL}_{2}(3)$ and there exists an involution $s \in C_{H}(r)$ inverting $x$ and $y$ and with $K\langle s\rangle \cong \mathrm{GL}_{2}(3)$.

Now consider the action of $y K\langle s\rangle$ on $\Omega(r)$. As $u^{t} \in \Delta_{1}^{2}(u), \operatorname{Mov}_{\Omega(r)}(u)=$ $\{a, b, c\}$ with $(a, b)$ a cycle of $t$ on $\Omega(r)$. As $K\langle s\rangle \cong \mathrm{GL}_{2}(3)$ is faithful on $\Omega(r), K$ is 2-transitive on the cycles of $t$ on $\Omega(r)$, so we may pick $s$ to have cycle $(a, b)$ and fix $c$ and $c t$. Thus $s$ inverts $y, u$, and $x$, so that (1) holds.

So by Lemma 5.6, $u^{t} \in \Delta_{3}^{2}(u)$. Let $X_{1}=Z\left(\left\langle u, u^{t}\right\rangle\right)$ and observe that $X_{1} \leq H_{y}$. Let $Y=N_{G}\left(X_{1}\right)^{\infty}$. By Lemma $4.7, Y / O_{5}(Y) \cong \mathrm{SL}_{2}(9)$, so $Y^{*}=$ $Y / O_{\{5,2\}}(Y)$ is faithfully represented as the alternating group on a set $\Gamma$ of order 6 with $\operatorname{Mov}_{\Gamma}(y)$ of order 3 and $\operatorname{Mov}_{\Gamma}(u)=\Gamma-\operatorname{Mov}_{\Gamma}(y)$. If $X_{1} \leq H_{x}$ then as $\langle y, x\rangle \cong \operatorname{SL}_{2}(3),\langle y, x\rangle$ induces $A_{4}$ on $\Gamma$, so $\left|\operatorname{Mov}_{\Gamma}(x) \cap \operatorname{Mov}_{\Gamma}(y)\right|=$ 2 , and hence $\left|\operatorname{Mov}_{\Gamma}(x) \cap \operatorname{Mov}_{\Gamma}(u)\right|=1$. Hence $\langle x, u\rangle$ induces $A_{5}$ on $\Gamma$, so by Lemma 5.3, $x \in \Delta_{2}^{2}(u)$. But then (3) holds.

So $X_{1} \npreceq H_{x}$. Thus $\operatorname{Fix}_{\Omega}(\langle x, y\rangle) \subseteq \operatorname{Fix}\left(\left\langle X_{1}, y\right\rangle\right)$, so the element $v \in H \cap$ $\Delta$ with $\operatorname{Mov}(v)=\operatorname{Fix}\left(\left\langle X_{1}, y\right\rangle\right)$ is contained in $C_{H}\left(\left\langle X_{1}, x, y\right\rangle\right)$. Let $S \in$ $\operatorname{Syl}_{2}\left(G_{y, v} \cap N\left(X_{1}\right)\right)$. By Lemma 3.7, $S v$ contains an involution $s$ inverting $u$. But $y X_{1} S \leq H_{y, v} \cap N\left(X_{1}\right)$ and some Sylow 2-subgroup of $H_{y, v} \cap N\left(X_{1}\right)$ normalizes $x$, so we may take $s \in G_{x, y, u}$. That is (2) holds.

Lemma 5.8. Let $p=x e y u$ be a path with $y \in \Delta_{2}^{2}(y)$ and $t \in\langle x, y\rangle$. Then

$$
H \cap x^{\perp} \cap\left(\Delta_{1}^{2}(u) \cup u^{\perp}\right) \cap\left(\Delta_{2}^{2}(y) \cup y^{\perp}\right) \neq \varnothing .
$$

Proof. If $u^{t} \in u^{\perp} \cup \Delta_{1}^{2}(u)$ then the lemma holds by Lemma 5.6(2). Therefore by Lemma $5.6(1)$, we may assume $u^{t} \in \Delta_{3}^{2}(u)$.

Let $X_{1}=Z\left(\left\langle u, u^{t}\right\rangle\right)$; again $X_{1} \leq H$. Let $Y=N_{G}\left(X_{1}\right)^{\infty}$. As in the 
proof of the previous lemma, $Y^{*}=Y / O_{\{5,2\}}(Y)$ is faithfully represented as the alternating group on a set $\Gamma$ of order 6 with $\operatorname{Mov}_{\Gamma}(y)$ of order 3 and $\operatorname{Mov}_{\Gamma}(u)=\Gamma-\operatorname{Mov}_{\Gamma}(y)$.

Suppose $X_{1} \leq H_{x}$. Then as $\langle x, y\rangle \cong \operatorname{SL}_{2}(5),\left|\operatorname{Mov}_{\Gamma}(x) \cap \operatorname{Mov}_{\Gamma}(y)\right|=1$, so $\left|\operatorname{Mov}_{\Gamma}(x) \cap \operatorname{Mov}_{\Gamma}(u)\right|=2$, and hence $x \in \Delta_{1}^{2}(u)$; so that the lemma holds.

Thus $X_{1} \Varangle H_{x}$, so $\operatorname{Fix}\left(\left\langle X_{1}, x, y\right\rangle\right)$ contains at least 2 points $a, b$, and hence we can pick $v \in H \cap \Delta$ with $\operatorname{Mov}(v)=\{a, b, c\}$ and $c \in \operatorname{Fix}(x) \cap$ $\operatorname{Mov}(y)$. Thus $v \in C\left(\left\langle X_{1}, x\right\rangle\right)$ and $\langle y, v\rangle \cong \mathrm{SL}_{2}(5)$.

As $\langle y, v\rangle \cong \operatorname{SL}_{2}(5),\left|\operatorname{Mov}_{\Gamma}(v) \cap \operatorname{Mov}_{\Gamma}(y)\right|=1$ and hence $\mid \operatorname{Mov}_{\Gamma}(v) \cap$ $\operatorname{Mov}_{\Gamma}(u) \mid=2$. Therefore $\langle v, u\rangle$ induces $A_{4}$ on $\Gamma$, so by Lemma 5.3, $v \in$ $\Delta_{1}^{2}(u)$.

Theorem 5.9. $\Delta$ has diameter 2 .

Before proving Theorem 5.9 we record one of its corollaries.

Theorem 5.10. $|G|=2^{8} \cdot 3^{7} \cdot 5^{6} \cdot 7 \cdot 11 \cdot 31 \cdot 37 \cdot 67$.

Proof. $|G|=\left|G_{x}\right||\Delta|$ and

$$
\left|G_{x}\right|=2^{8} \cdot 3^{7} \cdot 5^{3} \cdot 7 \cdot 11,
$$

so it remains to calculate $|\Delta|$. But by Theorem 5.9, $\Delta=\{x\} \cup \Delta(x) \cup \Delta^{2}(x)$, so by Lemmas 5.1 and 5.2 ,

$$
|\Delta|=9,606,125=5^{3} \cdot 31 \cdot 37 \cdot 67,
$$

completing the proof.

The remainder of this section is devoted to a proof of Theorem 5.9. We first show

Lemma 5.11. $\Delta$ is connected.

Proof. If not then the stabilizer $L$ of the connected component of $\Delta$ containing a member of $\Delta \cap H$ is of type $L y$ and hence contains $H$, has one class of involutions, and by induction on the order of $G$, has the order $n$ listed in Theorem 5.10. So $L$ is strongly embedded in $G$ and in particular by a standard result (cf. Exercise 16.5 in [1]) there is a subgroup $D$ of $L$ of odd order transitive on the involutions of $L$. This is a contradiction. For example as $D$ is of odd order, $D$ is solvable and hence has a Hall $\{3,67\}$-subgroup $K$. As $L$ has $n /|H|$ involutions, $|K|=3^{k} \cdot 67$ with $3 \leq k \leq 7$, so $K$ has an element of order 3.67 , contrary to Lemmas $4.5(4), 4.3$, and 4.6 .

Let $p=y_{0} y_{1} y_{2} y_{3}$ be a path in $\Delta$ of length 3 ; by Lemma 5.11 , it suffices to show $d\left(y_{0}, y_{3}\right) \leq 2$. Assume otherwise. Then $y_{2} \in \Delta_{i}^{2}\left(y_{0}\right)$ and $y_{1} \in \Delta_{j}^{2}\left(y_{3}\right)$ for some $i, j$. Let $x=y_{0}, y=y_{2}$, and $u=y_{3}$. We first observe

Lemma 5.12. We can choose $y_{1}$ and $y_{2}$ so that $i$ or $j$ is not 3 .

Proof. Suppose $y \in \Delta_{3}^{2}(x)$. Then by Lemma 5.1, $\Delta(x, y)=\Delta \cap\left\langle y_{1}, v\right\rangle$ and $v \in \Delta(x, y) \cap \Delta_{3}^{2}\left(y_{1}\right)$. Then by Theorem 3.10, there exists $d \in \Delta(x, y)$ such that $d \notin \Delta_{3}^{2}(u)$. Pick $y_{1}=d$ to insure $j \neq 3$. 
By Lemma 5.12 and reversing the roles of $x$ and $u$ if necessary, we may assume $i=1$ or 2. By Lemma 5.5, $\left|G_{x, u}\right|$ is odd, while by hypothesis $u \notin$ $\Delta^{2}(x)$. Thus $i \neq 1$ by Lemma 5.7 , so $i=2$. Then by Lemma 5.8 there exist $v \in \Delta(x) \cap \Delta_{1}^{2}(u)$. But now replacing $p$ by the path $u w v x$ for some $w \in \Delta(u, v)$, we reduce to the case $i=1$, which we have already handled.

This completes the proof of Theorem 5.9.

\section{THE COMMUTING GRAPH ON SUBGROUPS OF Ly OF ORDER 3}

In this section we continue the hypothesis and notation of $\S \S 4$ and 5 . In addition let $\Lambda$ be the commuting graph on subgroups of $G$ of order 3; we also write $\Lambda$ for the vertices of this graph. Thus $\Delta$ is a subgraph of $\Lambda$.

Lemma 6.1. (1) $\Delta$ and $\Lambda-\Delta$ are the orbits of $G$ on $\Lambda$.

(2) For each $a \in \Lambda-\Delta$ there is a subset $\hat{\phi}(a)$ of $\Delta$ of order 2 invariant under $G_{a}$ such that $a^{\perp} \subseteq x^{\perp}$ for each $x \in \hat{\phi}(a)$.

(3) If $a \in \Lambda-\Delta$ and $x, y \in \Lambda(a) \cap \Delta$ then $y \notin \Delta_{3}^{2}(x)$.

Proof. Part (1) is Lemma 4.5(4). Parts (2) and (3) follow from Lemma 4.6.

For each $a \in \Lambda-\Delta$, pick a member $\phi(a) \in \hat{\phi}(a)$. For $x \in \Delta$, let $\phi(x)=x$. Then by Lemma 6.1 ,

Lemma 6.2. (1) $\phi: \Lambda \rightarrow \Delta$ is a morphism of graphs.

(2) $\phi \circ l=\mathrm{id}_{\Delta}$, where $l: \Delta \rightarrow \Lambda$ is the inclusion.

(3) For each $a \in \Lambda, a^{\perp} \subseteq \phi(a)^{\perp}$.

Lemma 6.3. (1) $\mathscr{C}_{4}(\Lambda) \cap P(\Delta) \subseteq \mathscr{C}_{4}(\Delta)$.

(2) If $S$ is a set of cycles of $\Lambda$ with $\phi(S) \subseteq \mathscr{C}_{n}(\Delta)$ then $P(\Delta) \cap\langle S\rangle \subseteq \mathscr{C}_{n}(\Delta)$.

Proof. This follows from Lemmas 1.2, 1.3, and 6.2.

Lemma 6.4. Let $p=y_{0} \cdots y_{4}$ be a square in $\Delta$ such that $y_{2} \in \Delta_{i}^{2}\left(y_{0}\right)$ for $i=1$ or 2. Then $p \in \mathscr{C}_{3}(\Delta)$.

Proof. Let $t$ be the involution in $L=\left\langle y_{0}, y_{2}\right\rangle$. Then $\Delta\left(y_{0}, y_{2}\right)$ consists of those $y \in H \cap \Delta$ such that $\operatorname{Mov}(y) \subseteq \operatorname{Fix}(L)$. In particular if $i=1$ then $\Delta\left(y_{0}, y_{2}\right)$ is connected, while if $i=2$ then the graph on $\Delta\left(y_{0}, y_{2}\right)$ obtained by joining $x$ to $y$ if $x \in y^{\perp} \cup \Delta_{1}^{2}(y)$ is connected. Thus Lemma 1.4 completes the proof.

Lemma 6.5. Let $p=a_{0} \cdots a_{4}$ be a square in $\Lambda$. Then

(1) If $a_{i} \notin \Delta$ for some $i$ then $\phi(p) \in \mathscr{C}_{3}(\Delta)$.

(2) If $p \subseteq C_{G}(t)$ for some involution $t$ then $\phi(p) \in \mathscr{C}_{3}(\Delta)$.

Proof. If $a_{1} \notin \Delta$ then by Lemma 6.1(3), $\phi\left(a_{0}\right) \notin \Delta_{3}^{2}\left(\phi\left(a_{2}\right)\right)$, so $\phi(p) \in \mathscr{C}_{3}(\Delta)$ by Lemma 6.4 . Hence (1) holds. Further in proving (2), by (1) we may assume $p \subseteq \Delta$. Then as $p \subseteq C_{G}(t), a_{2} \notin \Delta_{3}^{2}\left(a_{0}\right)$, so again Lemma 6.4 completes the proof.

Define a path $p=a_{0} \cdots a_{n}$ in $\Lambda$ to be alternating if for all $i$ such that $a_{i} \notin \Delta, a_{i+1} \in \Delta$. 
Lemma 6.6. If $p$ is an alternating cycle in $C_{\Lambda}(t)$ then $\phi(p) \in \mathscr{C}_{3}(\Delta)$.

Proof. Write $\sim, \simeq$ for the invariant relation generated by triangles on $\Lambda, \Delta$, respectively. Let $p=a_{0} \cdots a_{n}$. We induct on the number $m$ of indices $i$ such that $a_{i} \notin \Delta$. If $m=0$ the lemma holds by Lemma 2.2(1). So let $a_{i} \notin \Delta$. Then as $p$ is alternating, $a_{i-1}$ and $a_{i+1}$ are in $\Delta$. Let $c$ be a cycle of $a_{i}$ on $\Omega$ and $x \in \Delta$ with $x^{*}=c^{*}$. Then $x \in \Delta\left(a_{i-1}, a_{i+1}\right)$ so $p \sim q=a_{0} \cdots a_{i-1} x a_{i-1} \cdots a_{n}$. Hence by Lemma 1.2(3), $\phi(p) \simeq \phi(q)$. But by induction on $m, \phi(q) \simeq 1$, so the proof is complete.

Lemma 6.7. Let $M_{11} \cong K \leq H$ and $a, b \in K \cap \Lambda$. Then

(1) There exists a path $p=a_{0} \cdots a_{2 n}$ in $H \cap \Lambda$ from a to $b$ such that for all $i, a_{2 i} \leq K$ and $a_{2 i+1} \in \Delta$.

(2) If $x \in \Delta$ with $a, b, t \in G_{x}$ then there exists an alternating path $p$ in $H \cap \Lambda$ from $a$ to $b$ such that $q=p b x a \in \mathscr{C}_{4}(\Lambda)$ and $\phi(q) \in \mathscr{C}_{3}(\Delta)$.

Proof. Part (1) is Lemma 2.2(2). So assume the hypotheses of (2). If $x \leq$ $H$ then $p=a x b$ works, so assume $t$ inverts $x$. Then $t$ induces an outer automorphism on $C_{G}(x) / x$, so by Lemma 3.1(3), $C_{G}(x\langle t\rangle) \cong M_{11}$. Thus we take $K=C_{G}(x\langle t\rangle)$ and apply (1) to get a path $p=a_{0} \cdots a_{2 n}$. Let $q=p b x a$. Now $q_{i}=x a_{2 i} a_{2 i+1} a_{2(i+1)} x$ is a square for each $i$, and as $a_{2 i} \notin \Delta, \phi\left(q_{i}\right) \in$ $\mathscr{C}_{3}(\Delta)$ by Lemma $6.5(1)$. Hence as $q$ is in the closure of the cycles $q_{i},(2)$ holds.

Lemma 6.8. If $p=y_{0} \cdots y_{4}$ is a square in $\Delta$ such that $y_{2} \in \Delta_{3}^{2}\left(y_{0}\right)$. Then $p \in \mathscr{C}_{3}(\Delta)$.

Proof. We first prove

(a) If $t \in G_{y_{i}}$ for each $i$, then the lemma holds.

For by Lemma 5.4(1), either $p \subseteq H$ or $t$ inverts each $y_{i}$, and by Lemma 6.5(2) we may assume the latter. Now by Lemma 4.6, $O_{3}\left(G_{y_{i}, y_{i}+1}\right)$ is of class 2 and hence not inverted by $t$, so there exists $a_{i} \in \Lambda\left(y_{i}, y_{i+1}\right) \cap H$. Pick a path $p_{i}$ from $a_{i}$ to $a_{i+1}$ as in Lemma 6.7(2) and let $q_{i}=p_{i} a_{i+1} y_{i} a_{i}$. Observe that $p$ is in the closure of the cycles $q_{i}$ and triangles $y_{i} y_{i+1} a_{i} y_{i}, 0 \leq i \leq 3$, and the cycle $q=p_{0} \cdots p_{3}$. So as $\phi\left(q_{i}\right) \in \mathscr{C}_{3}(\Delta)$ by the choice of Lemma $6.7(2)$, it remains by Lemma 6.3(2) to show $\phi(q) \in \mathscr{C}_{3}(\Delta)$. But this follows from Lemma 6.6.

So (a) is established. Now to the general case. Let $x=y_{0}, u=y_{1}, y=y_{2}$, and $v=y_{3}$. By Lemmas 3.3 and 3.7, $D=G_{x, u, y} \cong D_{30}$ with all involutions in $D$ inverting $x, y, u$, while by Lemma $5.1,\langle\Delta(x, y)\rangle=L \cong \mathbf{Z}_{3} / 5^{1+2}$. Let $Q=O_{5}(L)$ and $X=Z(Q)$, so that also $X=Z(L)$ and $D=X u\langle t\rangle$ for any involution $t \in D$. We next observe that

(b) 16 members of $\Delta(x, y)$ are inverted by involutions in $D$.

For $t$ acts on $L$ and inverts $u$, so $C_{Q}(t) \cong \mathbf{Z}_{5}$ is regular on the members of $\Delta(x, y)$ inverted by $t$. Further $t$ and $s$ invert the same $w \neq u$ if and only if $t s \in C_{D}(w)=X$.

Similarly there are 16 members of $\Delta(x, y)$ inverted by involutions in $G_{x, y, v}$, so there exist $w \in \Delta(x, y)$ inverted by involutions $t \in D$ and $s \in G_{x, y, v}$. 
Hence by (a), $x v y w x$ and $x w y u x$ are in $\mathscr{C}_{3}(\Delta)$, so $p$ is too.

Lemma 6.9. $\mathscr{C}_{4}(\Delta)=\mathscr{C}_{3}(\Delta)$.

Proof. This is immediate from Lemmas 6.4 and 6.8 .

Lemma 6.10. Let $x \in \Delta, y \in \Delta^{2}(x)$, and $s \in G_{x, y}$ an involution. Then either $s$ fixes a member of $\Delta(x, y)$ or $y \in \Delta_{2}^{2}(x)$ and $s$ centralizes a member of $\Lambda(x, y)$.

Proof. If $t \in\langle x, y\rangle$ then we check the assertion via the action of $\langle x, y, s\rangle$ on $\Omega$; the exceptional case occurs when $s$ centralizes $x$ and inverts $y$ or viseversa. So assume $y \in \Delta_{3}^{2}(x)$. Then $s$ acts on the 25 points of $\Delta(x, y)$, so as 25 is odd, $s$ fixes some member.

Lemma 6.11. If $p=y_{0} \cdots y_{5}$ is a pentagon in $\Delta$ such that $G_{y_{0}, y_{2}, y_{3}}$ has even order then $p \in \mathscr{C}_{3}(\Delta)$.

Proof. The proof is much like that of statement (a) of Lemma 6.8. Let $\sim$ be the invariant relation on paths of $\Lambda$ generated by triangles and squares. By Lemmas 6.3 and 6.9 it suffices to prove $p \sim 1$, or that $p$ is in the closure of cycles $q$ of $\Lambda$ with $\phi(q) \in \mathscr{C}_{4}(\Delta)$.

Let $x=y_{0}, y=y_{2}$, and $u=y_{3}$; without loss $t \in G_{x, y, u}$. By Lemma 6.10, there exist $a \in \Lambda(x, y)$ fixed by $t$ and either $a \in \Delta$ or $[t, a]=1$. Similarly there is $b \in \Lambda(x, u)$ with the same properties. As $p^{\prime}=x a y u b x \sim p$, it suffices to show $\phi\left(p^{\prime}\right) \in \mathscr{C}_{4}(\Delta)$. Let $b_{i}$ be the $i$ th term of $p^{\prime}$.

As in Lemma 6.8, $t$ centralizes some $a_{2} \in \Lambda(y, u)$. Similarly if $a \in \Delta$ then $t$ centralizes $a_{1} \in \Lambda(x, a)$, while if $a \notin \Delta$ we set $a_{1}=a$. We obtain $a_{i} \in \Lambda \cap C\left(\left\langle b_{i}, b_{i+1}, t\right\rangle\right)$ in an analogous manner. Pick a path $p_{i}$ from $a_{i}$ to $a_{i+1}$ as in Lemma 6.7(2) and let $q_{i}=p_{i} a_{i+1} b_{i} a_{i}$. Then $p^{\prime}$ is in the closure of triangles of $\Lambda$, the cycles $q_{i}, 0 \leq i \leq 4$, and $q=p_{0} \cdots p_{4}$. By the choice of $q_{i}, q_{i} \sim 1$ for each $i$, while by Lemma 6.6, $\phi(q) \in \mathscr{C}_{3}(\Delta)$. Thus the proof is complete.

\section{THE LYONS GRAPH IS SIMPLY CONNECTED}

In this section we continue the hypothesis and notation of $\S \S 4$ and 5 . We prove

Theorem 7.1. Let $G$ be a group of type Ly and $\Delta$ the commuting graph on the 3-central subgroups of $G$ of order 3 . Then $\Delta$ is simply connected.

We begin a short series of reductions. Let $\sim$ be the invariant relation on the paths of $\Delta$ generated by all triangles of $\Delta$. We must show each cycle in $\Delta$ is trivial with respect to $\sim$, so let $p=y_{0} \cdots y_{n}$ be a nontrivial cycle of minimal length $n$. By Theorem 5.9, $\Delta$ has diameter 2 , so by Lemma 3.3 in [2], $p$ is an $n$-gon and $n \leq 2 \operatorname{diam}(\Delta)+1=5$. Hence by Lemma $6.9, n=5$.

Let $x=y_{0}, y=y_{2}$, and $u=y_{3}$. By Lemma 6.11

Lemma 7.2. $G_{x, y, u}$ has odd order. 
Lemma 7.3. We may choose $p$ so that $y \in \Delta_{i}^{2}(x)$ for $i=1$ or 2 .

Proof. This is the same argument we used in Lemma 5.11. Namely if $y \in$ $\Delta_{3}^{2}(x)$ then by Lemma 5.1, $\Delta(x, y)=\Delta \cap\left\langle y_{1}, v\right\rangle$ for $v \in \Delta(x, y)-\left\{y_{1}\right\}$ and $v \in \Delta_{3}^{2}\left(y_{1}\right)$. Then by Theorem 3.10, there exists $d \in \Delta(x, y)$ with $d \notin \Delta_{3}^{2}(u)$. Hence as $x d y u y_{4} x \sim p$, we may assume $d=y_{1}$ and then shift the labeling of $p$ by 1 .

From now on we use Lemma 7.3 to assume $y \in \Delta_{i}^{2}(x)$ for $i=1$ or 2; without loss $t$ is the involution in $\langle x, y\rangle$. By Lemma 1.5

Lemma 7.4. $x^{\perp} \cap y^{\perp} \cap u^{\perp}=\varnothing$.

Lemma 7.5. $y \notin \Delta_{1}^{2}(x)$.

Proof. If so by Lemmas 5.7, 72, and 7.4, $u \in \Delta_{2}^{2}(x)$ and $u^{t} \in \Delta_{3}^{2}(u)$. Similarly

$$
y_{4}^{t} \in \Delta_{3}^{2}\left(y_{4}\right) \text {. }
$$

Let $s$ be the involution in $\langle x, u\rangle$; then by Lemma $5.1,\langle\Delta(x, u)\rangle /\langle s\rangle$ is an $A_{6}$-subgroup of $C_{G}(\langle x, s\rangle)$, so by Lemma 3.9(7) there is $v \in \Delta(x, u)$ such that

$$
v^{t} \notin \Delta_{3}^{2}(v)
$$

Now as $x y_{1}$ yuv $\sim p$, we may assume $v=y_{4}$. But then $(*)$ and $(* *)$ supply a contradiction.

By Lemma 7.5, $i=2$, so $y \in \Delta_{2}^{2}(x)$. Then by Lemma 5.8

Lemma 7.6. There exists $v \in H \cap x^{\perp} \cap\left(\Delta_{1}^{2}(u) \cup u^{\perp}\right) \cap\left(\Delta_{2}^{2}(y) \cup y^{\perp}\right)$.

Let $w \in v^{\perp} \cap u^{\perp}$ and $u_{1} \in y^{\perp} \cap v^{\perp}$. Then as $v \in \Delta_{1}^{2}(u) \cup u^{\perp}, q_{1}=x v w u y_{4} x$ and $q_{2}=v w u y u_{1} v$ are trivial by Lemma 7.5. Further $q_{3}=x y_{1} y u_{1} v x$ is trivial by Lemma 7.2 as $t \in G_{x, y, v}$. So as $p$ is in the closure of $q_{j}, 1 \leq j \leq 3, p$ is trivial.

Thus the proof of Theorem 7.1 is complete.

\section{THE UNIQUENESS OF GROUPS OF TYPE $L y$}

In this section we complete the proof of the main theorem. Thus we continue to assume the hypothesis and notation of $\S \S 4$ and 5. In addition let $x=\left\langle x_{1}\right\rangle$ and $y=\left\langle x_{2}\right\rangle$. By Lemma 4.4 there is $T \in \operatorname{Syl}_{3}(G)$ with $T \leq G_{x, y}$. Let $\Delta_{K}=\Delta \cap T, A=\left\langle\Delta_{K}\right\rangle, K=N_{G}(A), I=K_{x}$, and $\mathscr{U}=\left(G, K, \Delta, \Delta_{K}\right)$.

We prove the main theorem by appealing to the corollary to Theorem 1 of [2]. In particular the reader is referred to [2] for the definition of various concepts like uniqueness system, etc.

Lemma 8.1. $\mathscr{U}$ is a uniqueness system.

Proof. By Lemma 5.1, $\Delta(x)$ is an orbit under $G_{x}$, so $G$ is an edge transitive group of automorphisms of $\Delta$. 
Observe $G_{0}=\left\langle K, G_{x}\right\rangle$ is also of type $L y$, as $H=\left\langle H \cap K, H_{x}\right\rangle$ and $t$ is not weakly closed in $H_{x}$ with respect to $G_{x}$. Thus by Theorem $5.10,|G|=\left|G_{0}\right|$, so $G=G_{0}$. Similarly $G_{x}=\left\langle G_{x, y}, K_{x}\right\rangle$ by Lemma 3.1 and $K=\left\langle K(\{x, y\}), K_{x}\right\rangle$ as $K$ is 2-transitive on $\Delta_{K}$. Thus we have verified that $\mathscr{U}$ is a uniqueness system.

Assume $\bar{G}$ is a second group of type $L y$ with subgroup $\bar{K}$ and graph $\bar{\Delta}$, etc. By Lemma 8.1 applied to $\bar{G}$ we have a uniqueness system $\left(\bar{G}, \bar{K}, \bar{\Delta}, \bar{\Delta}_{\bar{K}}\right)$. Further

Lemma 8.2. There exists an isomorphism $\zeta: K \rightarrow \bar{K}$ with $x \zeta=\bar{x}$ and $y \zeta=\bar{y}$.

Proof. By Lemma 4.4, $K$ is the split extension of $A$ by $\mathbf{Z}_{2} \times M_{11}$. Hence by Lemma 2.5, there is an isomorphism $\zeta: K \rightarrow \bar{K}$. Further $\Delta_{K}$ and $\bar{\Delta}_{\bar{K}}$ are the orbits of length 11 on points of $A$ and $\bar{A}$, respectively, so by 2-transitivity of $K$ on $\Delta_{K}$ we may pick $x \zeta=\bar{x}$ and $y \zeta=\bar{y}$.

Lemma 8.3. $I=\operatorname{Aut}(I)$.

Proof. We have just observed $K$ is the semidirect product defined in Lemma 2.5, so the lemma is just Lemma 2.5(3).

Lemma 8.4. There exists an isomorphism $\alpha: G_{x} \rightarrow \bar{G}_{\bar{x}}$ with $I \alpha=\bar{I}$ and $y \alpha=$ $\bar{y}$.

Proof. By Lemmas 4.3 and 4.5(5), $G_{x}$ is the split extension of the covering group of $M c$ by an involutory outer automorphism, so there exists an isomorphism $\alpha: G_{x} \rightarrow \bar{G}_{\bar{x}}$. Further $y$ is determined up to conjugation in $G_{x}$ by the isomorphism type of $G_{x, y}$, so we can choose $y \alpha=\bar{y}$.

We can now complete the proof of the main theorem. First we observe that the hypotheses of Theorem 5 of [2] are satisfied with $Z(x)=x$ with respect to the maps $\zeta$ and $\alpha$ constructed in Lemmas 8.2 and 8.4. Namely hypotheses (1) and (2) of Theorem 5 of [2] are visibly satisfied, while the remaining two hypotheses follow from Lemma 8.3. Thus we conclude from Theorem 5 of [2] that $\mathscr{U}$ and $\overline{\mathscr{U}}$ are similar.

Next as $T \in \operatorname{Syl}_{3}(K) \cap \operatorname{Syl}_{3}\left(G_{x}\right)$ and $Z\left(K_{x, y}\right)=1$, Lemma 1.1 of [2] establishes the hypothesis of Theorem 2 of [2], so that Theorem says $\mathscr{U}$ is equivalent to $\overline{\mathscr{U}}$.

By Theorem 7.1, $\Delta$ and $\bar{\Delta}$ are simply connected. Further by Lemma 3.6, each triangle of $\Delta$ is $G$-conjugate to a triangle of $\Delta_{K}$. Therefore the corollary to Theorem 1 of [2] completes the proof.

\section{THE CLIQUe COMPLEX}

In this section $\Lambda$ and $\Delta$ are graphs. The clique complex of $\Delta$ is the simplicial complex $K(\Delta)$ whose vertices are the vertices of $\Delta$ and whose simplices are the cliques of $\Delta$. Observe that $K$ is a functor from graphs to simplicial complexes where the simplicial map induced by a morphism $d: \Lambda \rightarrow \Delta$ of graphs is defined by $d\left(\left\{x_{1}, \ldots, x_{n}\right\}\right)=\left\{d\left(x_{1}\right), \ldots, d\left(x_{n}\right)\right\}$ for each simplex $\left\{x_{1}, \ldots, x_{n}\right\}$ of $K(\Lambda)$. 
Define two morphisms $d, e: \Lambda \rightarrow \Delta$ to be contiguous if the induced maps of complexes are contiguous; i.e., for all simplices $s$ of $K(\Lambda), d(s) \cup e(s)$ is a simplex of $K(\Delta)$. Write $d \simeq e$ to indicate $d$ is contiguous to $e$.

Recall that we also have a functor $K \mapsto|K|$ from simplicial complexes to topological spaces. Further if $d$ is contiguous to $e$ then $|d|$ is homotopy equivalent to $|e|$. Thus

Lemma 9.1. Let $d: \Lambda \rightarrow \Delta$ and $e: \Delta \rightarrow \Lambda$ be morphisms of graphs such that $e \circ d \simeq \mathrm{id}_{\Lambda}$ and $d \circ e \simeq \mathrm{id}_{\Delta}$. Then $|K(\Lambda)|$ has the same homotopy type as $|K(\Delta)|$, and hence $|K(\Lambda)|$ and $|K(\Delta)|$ have the same homology and fundamental group.

This leads us to define graphs $\Delta$ and $\Lambda$ to have the same homotopy type if $|K(\Lambda)|$ and $|K(\Delta)|$ have the same homotopy type. Further we define the homology of $\Delta$ to be the homology of $|K(\Delta)|$. Finally we recall that by Remark 5 in [2], $\Delta$ is simply connected if and only if $|K(\Delta)|$ is simply connected. Thus we can restate Lemma 9.1:

Lemma 9.2. Let $d: \Lambda \rightarrow \Delta$ and $e: \Delta \rightarrow \Lambda$ be morphisms of graphs with $e \circ d \simeq$ $\mathrm{id}_{\Lambda}$ and $d \circ e \simeq \mathrm{id}_{\Delta}$. Then $\Lambda$ and $\Delta$ have the same homotopy type and homology and $\Lambda$ is simply connected if and only if $\Delta$ is simply connected.

Lemma 9.3. If $d: \Lambda \rightarrow \Delta$ and $e: \Delta \rightarrow \Lambda$ are morphisms with $a^{\perp} \subseteq e(d(a))^{\perp}$ for all $a \in \Lambda$ then $e \circ d \simeq \mathrm{id}_{\Lambda}$.

Lemma 9.4. Let $\Delta$ be a subgraph of the graph $\Lambda$ and assume for each $a \in \Lambda-\Delta$ there is some $d(a) \in \Delta$ with $a^{\perp} \subseteq d(a)^{\perp}$. Then $\Delta$ and $\Lambda$ have the same homotopy type and homology and $\Lambda$ is simply connected if and only if $\Delta$ is simply connected.

Proof. Extend $d: \Lambda \rightarrow \Delta$ by defining $d(x)=x$ for $x \in \Delta$, and let $e: \Delta \rightarrow \Lambda$ be inclusion. Then if $x, y \in \Lambda$ with $x \perp y$, we have $y \in x^{\perp} \subseteq d(x)^{\perp}$, so $d(x) \in y^{\perp} \subseteq d(y)^{\perp}$, and hence $d$ is a morphism. Then by Lemma 9.3, $e \circ d \simeq \mathrm{id}_{\Lambda}$, while of course $d \circ e=\mathrm{id}_{\Delta}$, so the lemma follows from Lemma 9.2.

Recall that each poset $P$ may be viewed as a simplicial complex whose vertices are the members of $P$ and whose simplices are the chains in $P$. Define the clique geometry $\operatorname{sd}(\Delta)$ to be the geometry over $I=\{0, \ldots, d\}$ whose objects of type $i$ are the $i$-dimensional simplices of $K(\Delta)$ and with incidence equal to inclusion, where $d=\operatorname{dim}(K(\Delta))$. Observe

$$
K(\operatorname{sd}(\Delta))=\operatorname{sd}(K(\Delta)) \text {, }
$$

where $\operatorname{sd}(K)$ is the barycentric subdivision of $K$; that is, $\operatorname{sd}(K)$ is the simplicial complex of the poset of simplices of $K$ partially ordered by inclusion. Thus as $\operatorname{sd}(K)$ has the same homotopy type as $K$, we have

$\Delta$ and $\operatorname{sd}(\Delta)$ have the same homotopy type.

For example let $G$ be a finite group and $p$ a prime. The Quillen complex $\mathscr{A}_{p}(G)$ for $G$ at the prime $p$ is the simplicial complex of the poset of all nontrivial elementary abelian $p$-subgroups of $G$ partially ordered by inclusion (cf. [8]). Define $\mathscr{A}_{p}^{*}(G)$ to be the simplicial complex whose vertex set consists of the maximal members of $\mathscr{A}_{p}(G)$ and $s \subseteq \mathscr{A}_{p}^{*}(G)$ is a simplex if $\bigcap_{A \in S} A \neq 1$. 
Lemma 9.7. Let $G$ be a finite group, $p$ a prime, and $\Lambda$ the commuting graph on subgroups of $G$ of order $p$. Then $K(\Lambda), \mathscr{A}_{p}(G)$, and $\mathscr{A}_{p}^{*}(G)$ have the same homotopy type.

Proof. The fact that $K(\Lambda) \simeq \mathscr{A}_{p}(G)$ was observed independently by Alperin. This proof is a variant of Alperin's proof; our original proof had a hole.

Let $\mathscr{C}_{1} ; \mathscr{C}_{2}$ be the covers of $K(\Lambda) ; \mathscr{A}_{p}(G)$ consisting of all subcomplexes $K(A) ; \mathscr{A}_{p}(A), A \in \mathscr{A}_{p}^{*}(G)$, respectively. Both covers are connically contractible, (cf. [8]) so by a standard lemma (cf. [8]) the corresponding nerves $N\left(\mathscr{C}_{1}\right) ; N\left(\mathscr{C}_{2}\right)$ of the covers have the same homotopy type as $K(\Lambda) ; \mathscr{A}_{p}(G)$, respectively. Finally visibly the maps $A \mapsto K(A)$ and $A \mapsto \mathscr{A}_{p}(A)$ are isomorphisms of $\mathscr{A}_{p}^{*}(G)$ with $N\left(\mathscr{C}_{1}\right)$ and $N\left(\mathscr{C}_{2}\right)$, respectively.

Lemma 9.8. Assume $\Delta$ is a graph and $m$ an integer such that for all simplices $s$ for $K(\Delta)$ of dimension at least $m$, there exists a unique simplex $d(s)$ of dimension $n$ containing $s$, where $n=\operatorname{dim}(K(\Delta))$. Let $\Gamma$ be the geometry on $I=\{1, \ldots, m\}$ whose objects of type $i<m$ are the $i$-dimensional simplices of $K(\Delta)$, whose objects of type $m$ are the $n$-dimensional simplices, and with incidence equal to inclusion. Then $\Delta$ and $\Gamma$ have the same homotopy type. In particular $H_{i}(\Delta)=0$ for $i>m$.

Proof. Apply Lemma 9.4 to the pair $\operatorname{sd}(\Delta), \Gamma$.

We can now prove Theorem 2. Let $G$ be of type $L y, \Delta$ the commuting graph on 3-central subgroups of order $3, \Lambda$ the commuting graph on subgroups of $G$ of order 3, and $\Gamma$ the 3-local geometry of $G$. By Lemmas 6.2 and 9.4, $\Delta$ and $\Lambda$ have the same homotopy type. By Lemma 9.7, $\Lambda$ and $\mathscr{A}_{3}(G)$ have the same homotopy type. The geometry $\Gamma$ is isomorphic to the subgeometry of $\operatorname{sd}(\Delta)$ of all simplices of dimension $0,1,10$, and by Lemma 3.6, each simplex of dimension at least 2 is contained in a unique conjugate of $A$. Thus by Lemma 9.8, $\Delta$ and $\Gamma$ have the same homotopy type and $H_{i}(\Delta)=0$ for $i>2$.

By Theorem 7.1, $\Delta$ is simply connected, so the fundamental group of $K(\Delta)$ is trivial. Thus as $H_{1}(\Delta)$ is the abelianization of the fundamental group $H_{1}(\Delta)=$ 0 . Of course as $\Delta$ is connected, the reduced homology group $\widetilde{H}_{0}(\Delta)=0$. Now

$$
\chi(\Delta)=\sum_{i}(-1)^{i} \operatorname{dim}\left(C_{i}(\Delta)\right)=\sum_{i}(-1)^{i} \operatorname{dim}\left(H_{i}(\Delta)\right)=1+\operatorname{dim}\left(H_{2}(\Delta)\right),
$$

so $\operatorname{dim}\left(H_{2}(\Delta)\right)=\chi(\Delta)-1$.

Let $n=|\Delta|, k=|\Delta(x)|$, and $m=\left|A^{G}\right|$. Then we have $\operatorname{dim}\left(C_{0}(\Delta)\right)=n$ and $\operatorname{dim}\left(C_{1}(\Delta)\right)=n k / 2$. Further for $i \geq 2$, each simplex of dimension $i$ is contained in a unique conjugate of $A \cap \Delta$, so $\operatorname{dim}(K(\Delta))=10$ and

$$
\sum_{i=2}^{10}(-1)^{i} \operatorname{dim}\left(C_{i}(\Delta)\right)=-m\left(\sum_{j=3}^{11}(-1)^{j}\left(\begin{array}{c}
11 \\
j
\end{array}\right)\right)=45 m .
$$

Notice $m=2^{3} \cdot 5^{2} \cdot 7 \cdot n$ and $k=2^{3} \cdot 5^{2} \cdot 7 \cdot 11$. Thus

$$
\begin{aligned}
\operatorname{dim}\left(H_{2}(\Delta)\right) & =n\left(1-2^{2} \cdot 5^{2} \cdot 7 \cdot 11+2^{3} \cdot 3^{2} \cdot 5^{3} \cdot 7\right)-1 \\
& =55,301 \cdot n-1=531,228,318,624
\end{aligned}
$$

completing the proof. 


\section{REFERENCES}

1. M. Aschbacher, Finite group theory, Cambridge Univ. Press, Cambridge, 1986.

2. M. Aschbacher and Y. Segev, Extending morphisms of groups and graphs, to appear in Ann. Math.

3. R. Brauer and M. Suzuki, On finite groups of even order whose 2-Sylow subgroup is a quaternion group, Proc. Nat. Acad. Sci. U.S.A. 45 (1959), 1757-1759.

4. L. Finkelstein, The maximal subgroups of Conway's group $C_{3}$ and the McLaughlin's group, J. Algebra 25 (1973), 58-89.

5. G. James, The modular characters of the Mathieu groups, J. Algebra 27 (1973), 57-111.

6. Z. Janko and S. Wong, A characteristic of the McLaughlin's simple group, J. Algebra 20 (1972), 203-225.

7. R. Lyons, Evidence for a new finite simple group, J. Algebra 20 (1972), 540-569.

8. D. Quillen, Homotopy properties of the poset of nontrivial p-subgroups, Adv. in Math. 28 (1978), 101-128.

9. C. Sims, The existence and uniqueness of Lyons' group, Gainesville Conference on Finite Groups, North-Holland, Amsterdam, 1973, pp. 138-141.

10. J. Conway, et al., Atlas of finite groups, Clarendon Press, Oxford, 1985.

Department of Mathematics, California Institute of Technology, Pasadena, CaliFORNIA 91125

Department of Mathematics, Ben Gurion University, Beer Sheva 84105, Israel 